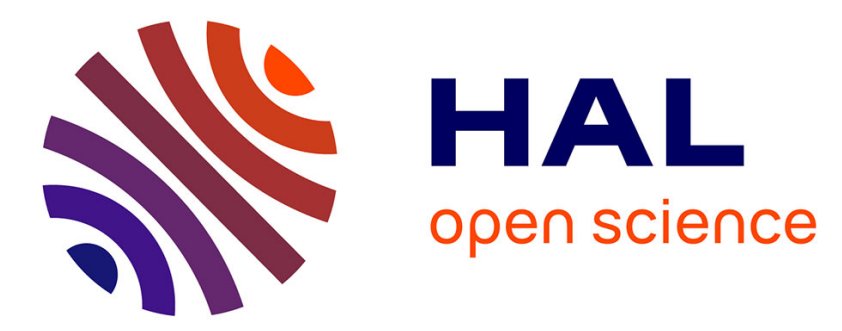

\title{
Experiments and numerical simulations of nonlinear vibration responses of an assembly with friction joints - Application on a test structure named "Harmony"
}

\author{
M Claeys, Jean-Jacques Sinou, J-P Lambelin, R Todeschini
}

\section{- To cite this version:}

M Claeys, Jean-Jacques Sinou, J-P Lambelin, R Todeschini. Experiments and numerical simulations of nonlinear vibration responses of an assembly with friction joints - Application on a test structure named "Harmony". Mechanical Systems and Signal Processing, 2016, 70-71, pp.1097 - 1116. 10.1016/j.ymssp.2015.08.024 . hal-03275347

\section{HAL Id: hal-03275347 \\ https://hal.science/hal-03275347}

Submitted on 1 Jul 2021

HAL is a multi-disciplinary open access archive for the deposit and dissemination of scientific research documents, whether they are published or not. The documents may come from teaching and research institutions in France or abroad, or from public or private research centers.
L'archive ouverte pluridisciplinaire HAL, est destinée au dépôt et à la diffusion de documents scientifiques de niveau recherche, publiés ou non, émanant des établissements d'enseignement et de recherche français ou étrangers, des laboratoires publics ou privés. 
M. Claeys, J-J. Sinou, J-P. Lambelin and R. Todeschini, Experiments and numerical simulations of nonlinear vibration responses of an assembly with friction joints - Application on a test structure named "Harmony", Mechanical Systems and Signal Processing, 70-71, 1097-116, 2016.

doi:10.1016/j.ymssp.2015.08.024

\title{
Experiments and numerical simulations of nonlinear vi- bration responses of an assembly with friction joints - Application to a test structure named "Harmony"
}

\author{
M. Claeys ${ }^{a, b}$, J-J. Sinou ${ }^{b, c}$, J-P. Lambelin ${ }^{a}$ and R. Todeschini ${ }^{a}$ \\ ${ }^{a}$ CEA/CESTA, CS60001, 15 av. des Sablières, 33116 Le Barp, France \\ ${ }^{b}$ Laboratoire de Tribologie et Dynamique des Systèmes UMR-CNRS 5513, Ecole Centrale de Lyon, \\ 36 avenue Guy de Collongue, 69134 Ecully Cedex, France (email: jean-jacques.sinou@ec-lyon.fr, tel: \\ $+33(0) 472186466$, fax: $+33(0) 472189144)$ \\ ${ }^{c}$ Institut Universitaire de France, 75005 Paris, France.
}

\begin{abstract}
In presence of friction, the frequency response function of a metallic assembly is strongly dependent on the excitation level. The local stick-slip behavior at the friction interfaces induces energy dissipation and local stiffness softening. These phenomena are studied both experimentally and numerically on a test structure named "Harmony".

Concerning more specifically the numerical part of the present paper, a classical complete methodology from the finite element and friction modeling to the prediction of the nonlinear vibrational response is implemented. The well-known Harmonic Balance Method with a specific condensation process on the nonlinear frictional elements is achieved.

Also, vibration experiments are performed to validate not only the finite element model of the test structure named "Harmony" at low excitation levels but also to investigate the nonlinear behavior of the system on several excitation levels. One of the original contributions of the present study is the use of the a scanning laser vibrometer to measure the nonlinear behavior and the local stick-slip movement near the contacts. It gives the opportunity to compare the predicted numerical nonlinear responses with the experimental ones.
\end{abstract}

\section{Introduction}

In the past, the vibration responses of industrial structures were mainly studied using a linear analysis. Indeed, numerical simulations for both modal analysis and Frequency Response Function of linear systems are implemented in every Finite Element software and are widely used in industry. However, 
experimentally, the frequency response of an assembly often appears to be strongly dependent on the excitation level which cannot be explained by a classical linear analysis. This nonlinear evolution may be due in particular to friction in the joints, large displacements, non-elastic materials or contacts $[1,2]$.

Therefore, the incorporation of nonlinear phenomena into complex mechanical systems currently gives rise to major problems during the design of industrial mechanical structures. It is clear that optimizing mechanical structures with respect to their vibration behavior requires a detailed understanding of the structures along with a highly refined model. Including the set of nonlinear elements that play a predominant role in the dynamic behavior of structures, proves essential not only to studying the dynamic behavior of systems, but also to devising robust and reliable system designs able to withstand the range of loadings potentially applied. So both an efficient modeling of the nonlinear behavior of mechanical systems and the development of nonlinear computational techniques are essential in order to proceed with an efficient and quick analysis of complex problems. It is known that various computational techniques in the treatment of nonlinear differential equations have been proposed in a wide range of mechanical engineering problems $[3,4,5]$. According to Sarrouy and Sinou [6] the Harmonic Balance Method is one of the most popular methods for approximating the stationary nonlinear responses of mechanical systems. The main idea of this numerical process is to approximate the nonlinear responses and the nonlinear forces in dynamical systems by their Fourier series. This method was implemented in a previous work of the authors [7] and compared with the method of multiple scales and the shooting method for the computation of the nonlinear response of nonlinear systems. It was illustrated that the Harmonic Balance Method gave excellent results in terms of both precision and computation time. This is the reason why this method has been chosen for the present study. In the recent years, many efforts have also been done to increase the numerical efficiency of this nonlinear approach for vibration damping by dry friction $[8,9,10,11,12,13]$. Among them, we can cite the Dynamic Lagrangian Frequency Time method (DLFT), proposed by Nacivet et al. [14] that enables to handle directly the non-smooth unilateral and frictional contact laws.

Also, conducting studies with experiments, modeling and numerical simulation seems necessary nowadays in order to better understand the limitation of modeling and to increase the confidence of the numerical models with nonlinear frictional elements. One of the primary objectives is also that the numerical model can be used to minimize the need for conducting expensive experiments for optimizing such mechanical structures. Some previous works have already presented this type of study that combines experimentation and simulation in the field of structures with friction joints. This is more specifically the case in the field of turbomachinery bladed disks for which significant vibration damping by friction forces can occur. Therefore the friction damping concept has been frequently applied in turbomachinery applications and various research works for analyzing the dynamic characteristics of structures with friction joints have been undertaken. For example we can mention researches on structures with underplatform dampers $[15,16,17,18,19]$, approach for characterizing the dynamic behavior of a friction damper [20], shrouds with a frictional interface to reduce the dynamic stresses in turbine blades via the comparison between measured and calculated frequency response functions for bending and torsional vibrations of the blade [21], optimization of interblade friction damper design with respect to an optimal damper geometry and damper mass[22], impact of friction in blade with root joints [23] or friction rings [24]. More generally, the review papers of Gaul and Nitsche [25] and 
Feeny et al. [26] provided also a historical overview of structural and mechanical systems on the general topic of friction damping.

In the present study, we propose to investigate the nonlinear behavior of a complex structure, "Harmony", which includes nonlinear frictional elements. Results from the nonlinear numerical simulations are compared with those from various experiments. Even if the primary objective of the present study is the validation of existing numerical methods, one of the main contributions is to be able to perform measurements of nonlinear vibrations near the contacts by using a scanning laser vibrometer, providing the opportunity to compare experiments with the numerical results.

The paper is divided into three parts. Firstly, a brief description of the test structure and the analysis of various experimental data from vibrational tests on a bench in the CEA laboratory are presented. Secondly, the paper focuses on the finite element modeling (linear elements, model reduction and frictional modeling) and the global updating procedure. The nonlinear simulation based on the well-known Harmonic Balance Method with a condensation process is then explained in Section 4. Finally, results from the nonlinear numerical simulations are compared with those from experiments. Multi-harmonic comparisons are performed [7] and a local analysis of the stick-slip movement is also proposed. This last part is one of the originality and peculiarities of the study that proposes to increase the confidence in the numerical models currently used for friction damping simulation by providing comparisons between experiments and simulations in the neighborhood of the contact interface.

\section{Experiments}

\subsection{Motivations}

In presence of friction, the Frequency Response Function (FRF) of an assembly evolves while increasing the excitation level. The resonance peaks may flatten or sharpen with the increase of the excitation level when the resonance frequencies are lowered. The objective of the experimental part of this study is to reproduce experimentally these phenomena on a structure where the origins of friction are obvious. This objective led to the design of a specific test structure named "Harmony". This proposed test structure includes four contact joints (identified a priori) where friction is expected at high excitation levels. "Harmony" is designed with a minimal number of joints so as to be able to limit the sources of nonlinear behaviors and energy dissipation. Therefore, it enables to fully understand the concept of modeling nonlinear friction connections and their impacts through experimental tests and comparisons with numerical simulation. Yet the structure is complex enough to demonstrate the ability of the proposed simulation method to be applied on real industrial structures with nonlinear friction connections. The experiments are performed in an industrial way, with an industrial shaker and accelerometers to measure experimental FRF. In addition, specific instrumentation is used to record the local stick-slip movement related to friction in a joint zone. A scanning laser vibrometer is thus additionally deployed. The two main substructures of the assembly are tested separately at low excitation level to be able to update a finite element model before the modeling of joints. The assembly is then tested with increasing excitation levels to observe the effect of friction. 

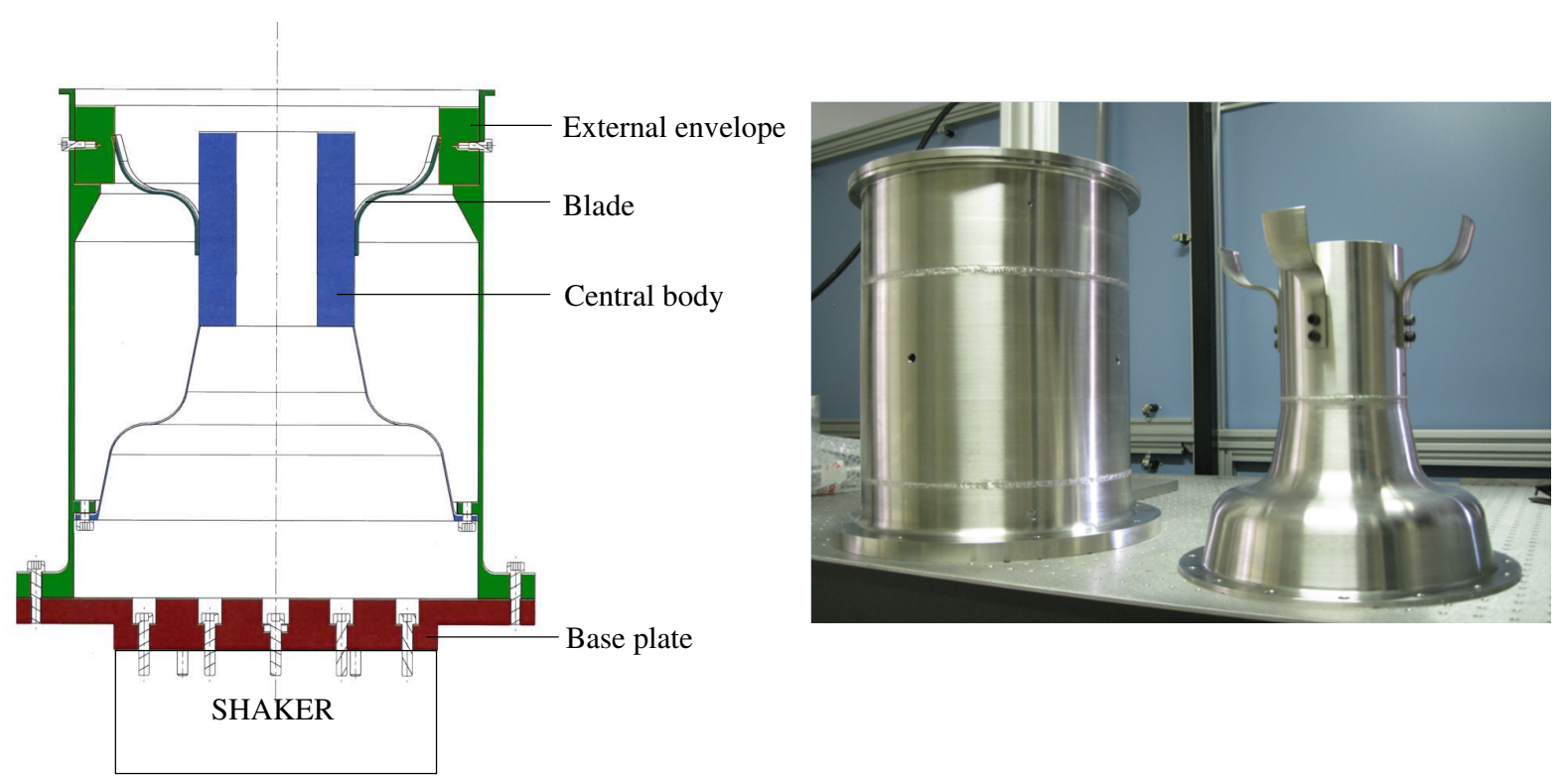

Figure 1: Cross-section schema (left) and picture (right) of the "Harmony" structure.

\begin{tabular}{|l|l|l|l|}
\hline Part & Material & $\begin{array}{l}\text { Dimensions }(\mathrm{mm}) \\
\text { Max radius, height }\end{array}$ & Mass (kg) \\
\hline Central Body & Stainless steel 304L & 160,300 & 11.92 \\
\hline External envelope & Stainless steel 304L & 204,420 & 44,88 \\
\hline Base plate & Aluminium 2017A & 204,40 & 9,47 \\
\hline Blade $(\mathrm{x} 4)$ & $\begin{array}{l}\text { High resistance steel } \\
\text { Z8-cnd17-04 }\end{array}$ & 65,118 & 0,1086 \\
\hline
\end{tabular}

Table 1: Parts characteristics.

\subsection{Experimental Set-Up}

The "Harmony" structure is presented in Fig. 1. The bottom of the central body is clamped to the external envelope and its top is in contact with this envelope through 4 blades. The characteristics of each part of the assembly are detailed in Table 1, and the mass values are measured. During the assembly, the central body is inserted inside the envelope from the bottom. The blades are compressed, so that contact is never broken, even during vibration experiments. This structure is designed to have a fundamental axial mode that stresses the contact joints between the 4 blades and the external envelope, initiating friction at high excitation levels. The structure is tested with the base clamped to a shaker. Swept sine experiments are realized in the vicinity of the first axial mode. Two sweep rates have been used. The general sweep rate is $10 \mathrm{~Hz} \cdot \mathrm{s}^{-1}$. At high excitation level, the sweep rate is reduced to $1 \mathrm{~Hz} . \mathrm{s}^{-1}$ in the frequency range where friction occurs. This low sweep rate minimizes transient phenomena. 10 three-dimensional accelerometers measure the structure response. 4 strain gages are placed under the blades. The location of these sensors is detailed in Fig. 2. The shaker is 

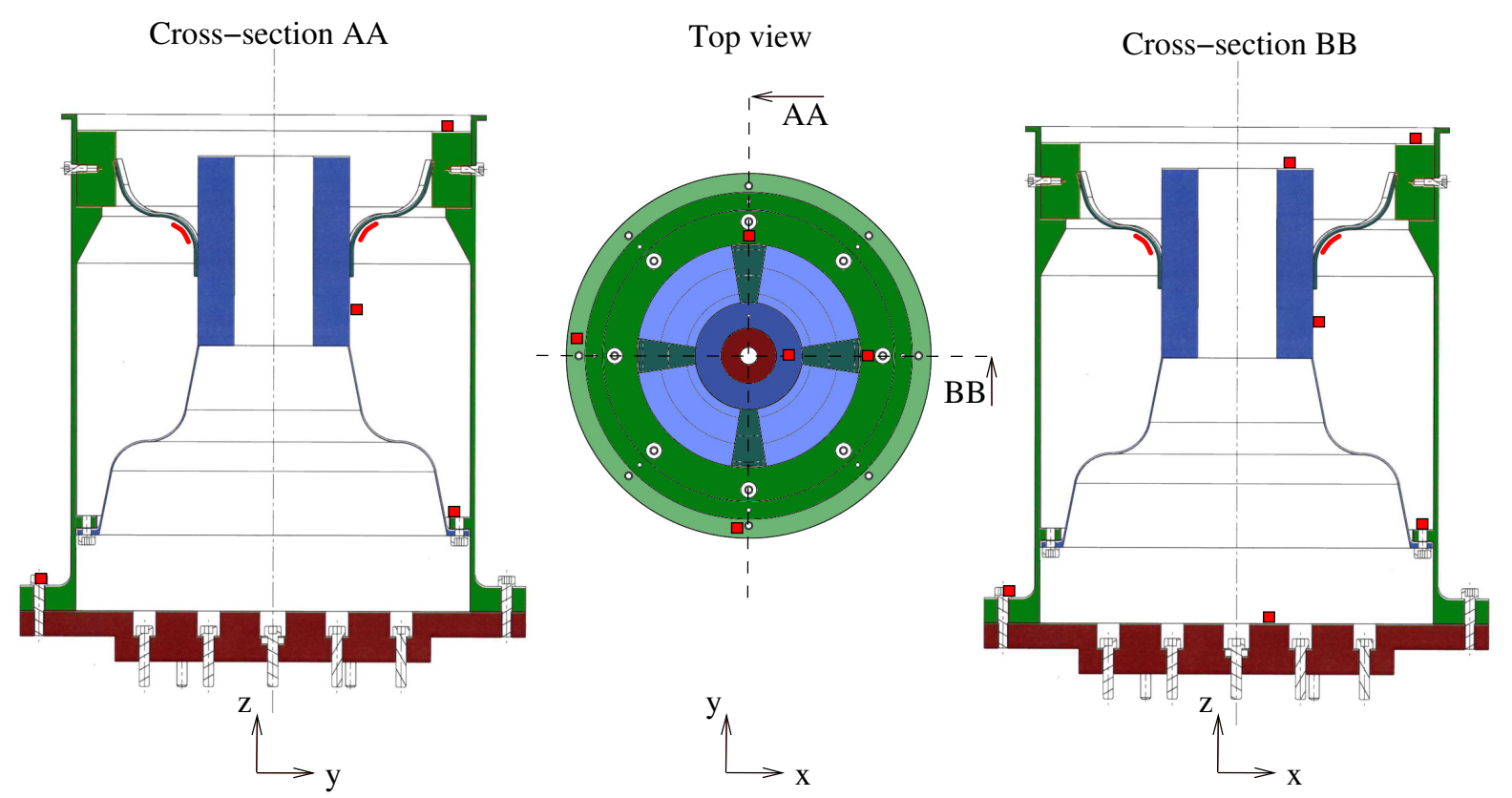

Figure 2: Location of the sensors. Accelerometers are represented with red squares. 4 strain gages, depicted in red, are placed under the blades.

piloted with a constant input tension amplitude.

\subsection{Substructure modal analysis}

Before the main tests on the assembly, the two main substructures -the central body with blades and the external envelope- are tested separately. The corresponding experimental set-ups are depicted in Fig. 3. The first vertical modes of these substructures are then used to update the finite element model of each substructure. The experimental eigenfrequencies are presented in Table 2 . For the tests on the substructure composed of the central body with blades, the strain gages' signal is unable to identify whether the resonance involves the blades and how. A mode where the blades and the body movements are synchronous in phase is identified at $218.7 \mathrm{~Hz}$. Then, between $247 \mathrm{~Hz}$ and $251.5 \mathrm{~Hz}$, several resonance peaks are observed. The 4 blades are not synchronous in phase in this frequency range, and it is difficult to identify separated modes.

\subsection{Frequency Response Functions}

The frequency responses of the "Harmony" assembly are recorded with the shaker piloted with a constant input tension amplitude. To be able to compare with the simulation, this input is modeled as a constant force input. Piloting the shaker with a force measurement was not possible in the experimental installation. In the narrow frequency range considered, the force amplitude is assumed to be independent from the excitation frequency. The amplitude of the force for each excitation level is identified. A first identification of the force has been realized at low excitation level. This comparison 

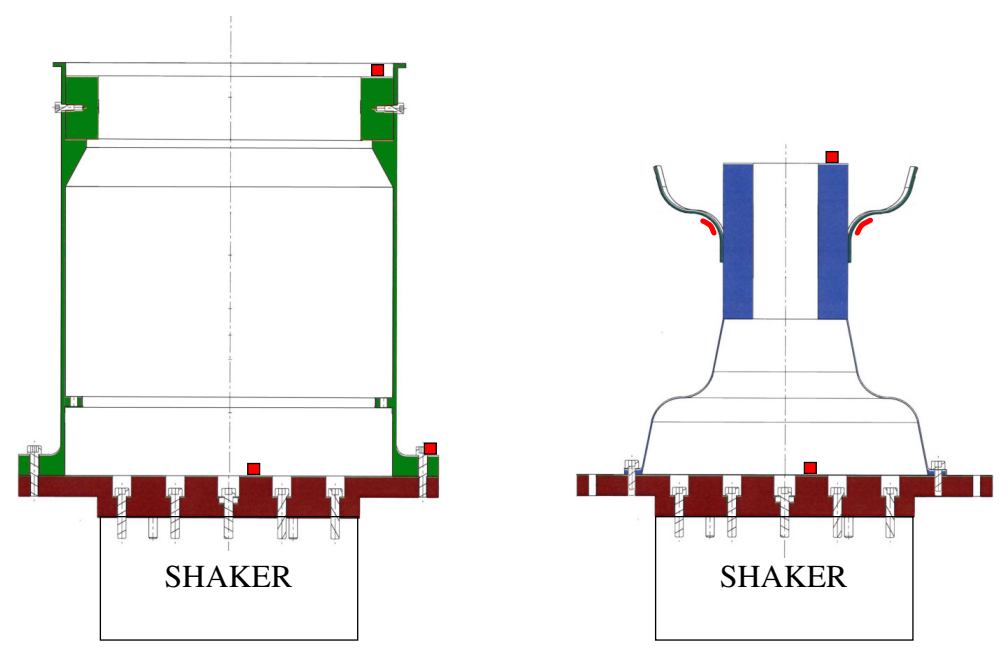

Figure 3: Schema of the substructure experimental set-ups.

\begin{tabular}{|l|l|l|}
\hline Substructure & Mode & Resonance frequency \\
\hline Substructure 1: Envelope & $1^{\text {st }}$ vertical mode & $889 \mathrm{~Hz}$ \\
\hline $\begin{array}{l}\text { Substructure 2: } \\
\text { Central Body + Blades }\end{array}$ & $\begin{array}{l}1^{\text {st }} \text { vertical mode (blades and } \\
\text { central body synchronous) }\end{array}$ & $218.7 \mathrm{~Hz}$ \\
\hline $\begin{array}{l}\text { Substructure 2: } \\
\text { Central Body + Blades }\end{array}$ & $\begin{array}{l}\text { Several modes involving blades } \\
\text { asynchronously }\end{array}$ & {$[247-251.5] \mathrm{Hz}$} \\
\hline
\end{tabular}

Table 2: Substructure eigenfrequencies.

will be presented in Section 3.2. Close to the resonance, the simulated results obtained with a $70 \mathrm{~N}$ excitation force fit the measurements obtained with a $20 \mathrm{mV}$ tension amplitude. At $100 \mathrm{~Hz}$, away from any resonance, the system behaves linearly. That is the reason why the amplitude at the response at $100 \mathrm{~Hz}$ is used to identify the excitation force. The method used is the following:

$$
\text { Excitation level }=70 \mathrm{~N} \cdot \frac{\text { Response level at } 100 \mathrm{~Hz}}{\text { Response level at } 100 \mathrm{~Hz} \text {, obtained with a } 70 \mathrm{~N} \text { excitation }}
$$

The Frequency Response Function (FRF) for a given physical point of the structure is the vertical response acceleration divided by the force excitation level. The obtained experimental FRF amplitudes with increasing excitation levels are plotted in Fig. 6 and Fig. 7. The friction phenomenon is characterized by an increasing dissipation and a decreasing resonance frequency. The effect of friction is obvious in the 2 different response points depicted in Fig. 6 and Fig. 7. The nonlinear behavior is observed both at the top of the central body (Fig. 6) and on the base plate (Fig. 7). This reveals that friction influences the global frequency response of the structure even if the phenomenon is local. For each FRF measurement, two successive tests have been performed in the same experimental setting to check the repeatability of the results obtained for a given configuration. In Fig. 6 and Fig. 7, it is remarkable that the variance of experimental results increases when friction occurs. The very low variance away from friction apparition $(<1 \%$ for sufficiently high response levels) characterizes the 


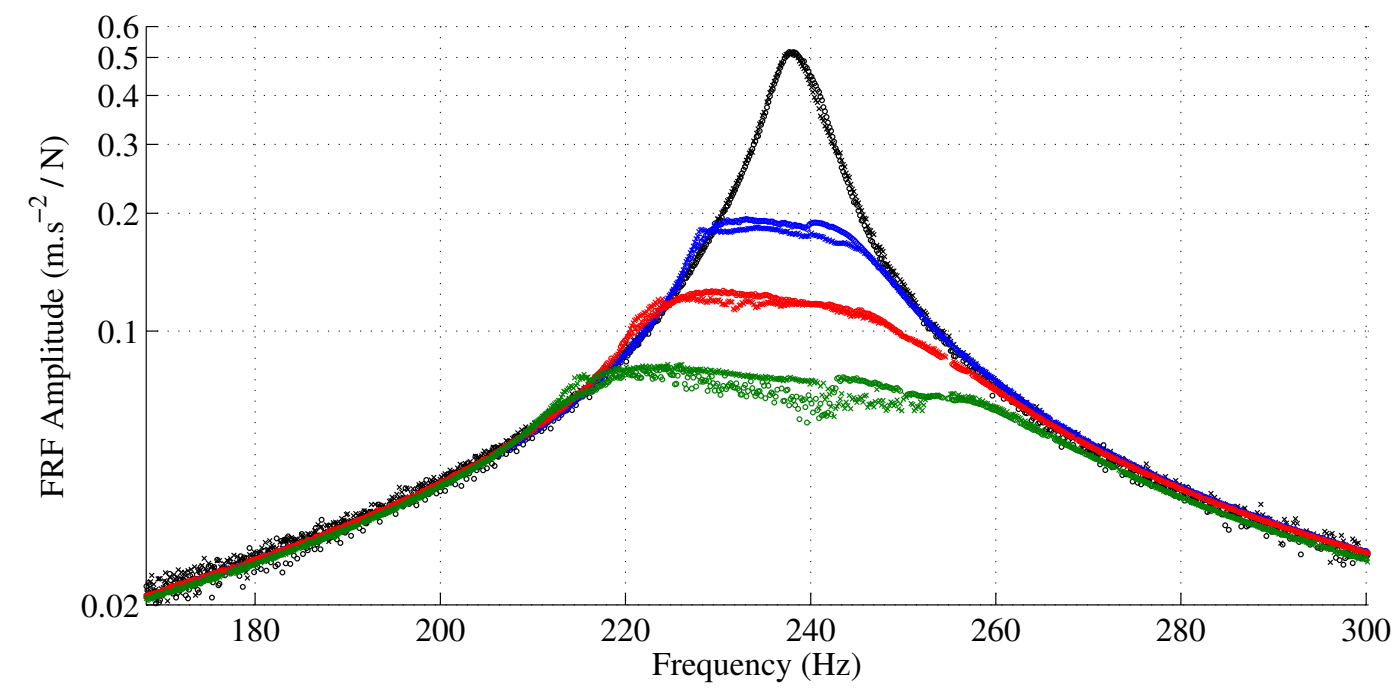

Figure 4: Experimental FRF at the top of the central body with increasing excitation level. The excitation levels are $70 \mathrm{~N}$ (black), 1070N (blue), 1875N (red) and 2910N (green). For each excitation level the first experimental results obtained are plotted with crosses and the second with circles.

measurement uncertainties. The higher variance in the friction frequency range (up to $30 \%$ ) reveals that the local behavior and the frictional mechanisms at junctions play a predominant role in test repeatability. However it is observed that, at all events, this variance is still low in comparison with the difference between two results with different excitation levels. A deterministic model method is thus relevant and sufficient for this study.

\subsection{Laser vibrometry}

The main objectives of the use of a scanning laser vibrometer for the present work are twofold. First, the possibility to observe friction through the deformation of the contact zones. Second, the opportunity to compare the predicted numerical results with experiments. This second objective will be explained and carried out in Section 5.3.

The laser vibrometer is positioned vertically above the structure. A $238 \mathrm{~Hz}$ fixed frequency sinusoidal excitation is applied, the stationary regime is observed. The 52 measurement points depicted in Fig. 8 are successively scanned by the vibrometer. In each point the instant vertical velocity is measured and recorded during a few periods $(50 \mathrm{kHz}$ sampling). Assuming the stationary regime, the different responses are then synchronized using the excitation signal phase. A video of the blade movement over an excitation period is thus obtained. This video is illustrated in Fig. 9. The 9 images correspond to a regular sampling over an excitation period. The color and the position of the points represent the instant velocity. A red and high position corresponds to a positive vertical velocity while a green and low position corresponds to a negative vertical velocity. When the colors of the top of the blade and the envelope are different, it reveals slipping. Otherwise the blade is stuck to the envelope. Fig. 9 thus enables an appreciation of the alternating stick-slip phenomenon that characterizes friction. The top 


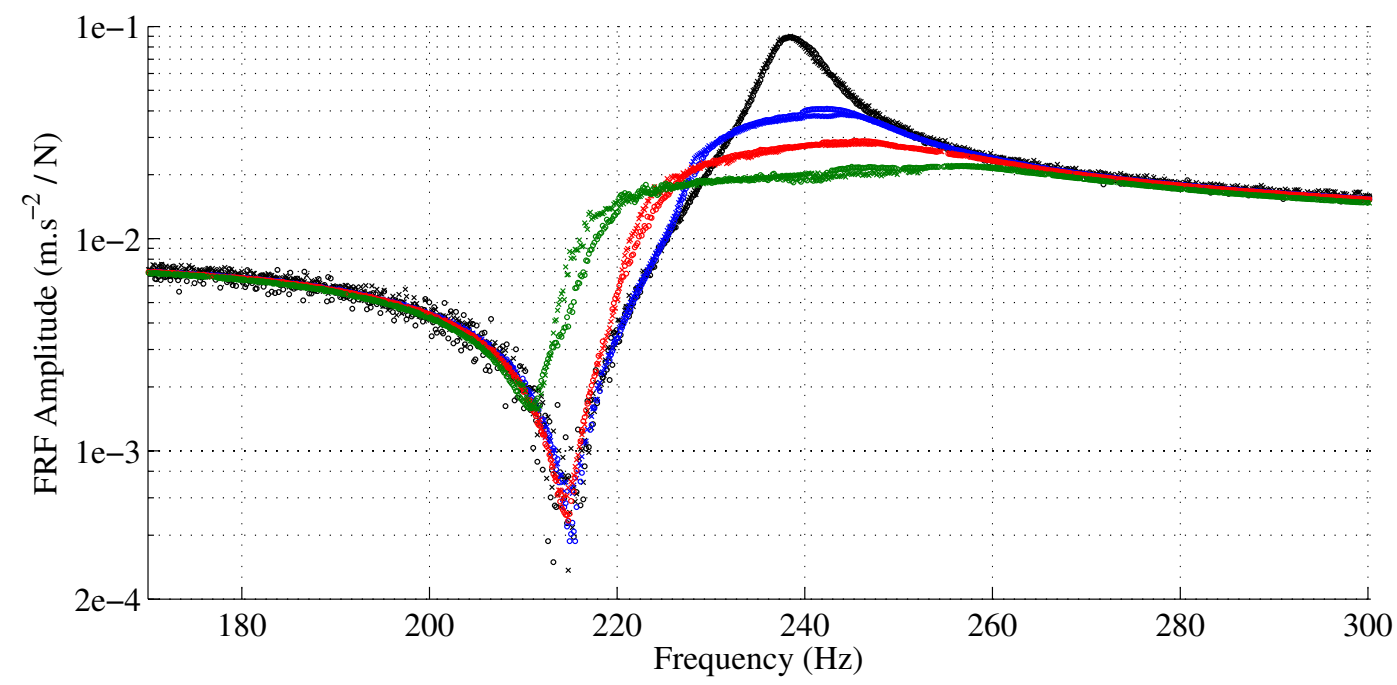

Figure 5: Experimental FRF on the base plate with increasing excitation level. The color legend is the same that in Fig. 6.

of the blade is deformed during slipping (frame 2, 3, 4, 8 and 9). Partial slipping happens in frame 2 . In Section 3, the top of the blade is assumed not to be deformable and partial slipping is not enabled. This assumption that simplifies calculations is not fully in agreement with the real local behavior.

\section{Modeling}

\subsection{Finite Element Model}

The structure is modeled with the finite element software Abaqus. Fig. 10 presents this model which includes solid, shell and thick shell elements, depicted respectively in green, yellow and grey. The excitation force is applied at the bottom of the moving part of the shaker. In the frequency range considered $([170-300] \mathrm{Hz})$, the shaker is modeled by an inertial mass whose transverse and rotational degrees of freedom are blocked. This mass is connected to the bottom of the base plate through a structural coupling. A soft spring $\left(K_{\text {soft }}=22800 \mathrm{~N}\right.$.m according to the vibrator manufacturer $)$ links this mass to the ground.

Each contact zone at the top of a blade is controlled by a unique reference node, named "Contact Zone", depicted in Fig. 10. All the degrees of freedom of the 10 nodes of this contact zone are rigidly controlled by this reference node. In the same way, the 11 nodes of the upper edge of the blade are rigidly driven by the reference node named "Top_Blade". The contact is modeled by a strong stiffness connector $\left(10^{10} \mathrm{~N} . \mathrm{m}^{-1}\right.$ for each component) between these two reference nodes. Vertical slipping may then be simply described as the cancellation of the vertical component of this connector. At this step, in this linear model, all the blades remain stuck to the envelope. This modeling is in agreement with the very low excitation level experiments where friction is not activated. The same kind of modeling 


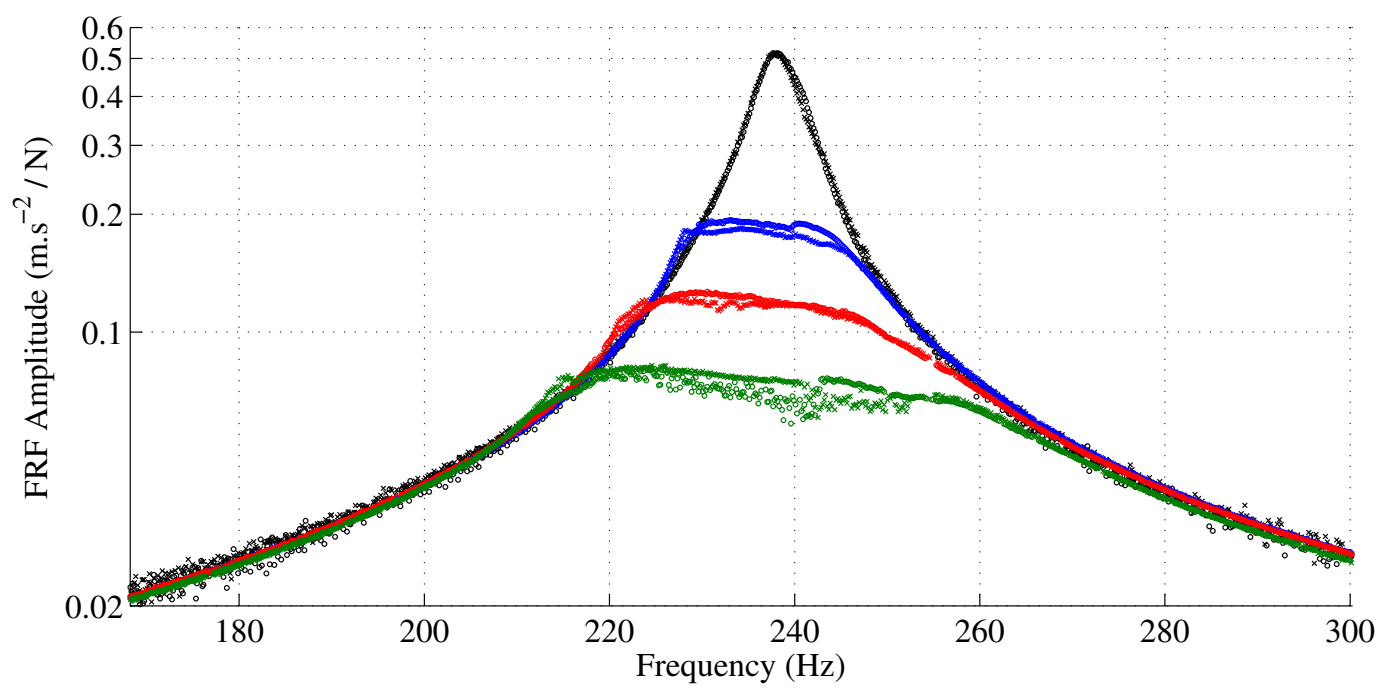

Figure 6: Experimental FRF at the top of the central body with increasing excitation level. The excitation levels are $70 \mathrm{~N}$ (black), 1070N (blue), 1875N (red) and 2910N (green). For each excitation level the first experimental results obtained are plotted with crosses and the second with circles.

\begin{tabular}{|l|l|l|}
\hline Instance & Numerical mass & Error with experiments \\
\hline Base plate & $9.57 \mathrm{~kg}$ & $1 \%$ \\
\hline Envelope & $44.79 \mathrm{~kg}$ & $0.2 \%$ \\
\hline Central body & $11.92 \mathrm{~kg}$ & $<0.1 \%$ \\
\hline Blade & $108.810^{-3} \mathrm{~kg}$ & $0.2 \%$ \\
\hline
\end{tabular}

Table 3: Instances' model mass and comparison with measurements.

with connectors and reference nodes is used to model the joint between the blade and the central body. These two instances are connected through 2 screws. The screws are modeled using strong stiffness connectors between 2 reference nodes (named "Screw_1" and "Screw_2"). Each reference node drives the movement of an influence zone of radius $0.01 \mathrm{~m}$. The influence zones on the blade are depicted in red in Fig. 10. In mirror, the same influence zones are defined on the central body.

With this model, friction is localized in the vertical component of the connector between the points "Top_Blade" and "Contact_Zone". The nonlinear contact model can then be a 1 dimension model. This modeling significantly simplifies nonlinear simulations but assumes that the top of the blade is not deformable which is not truly in agreement with vibrometry observation (see Section 2.5).

\subsection{Modal analysis and model validation}

First, the finite element instances' mass of the assembly is compared with the measured mass. Table 3 presents this comparison. The higher error, in the base plate, is due to the threaded holes that are not modeled. The modeling of the central body and the blades is validated with the substructure 


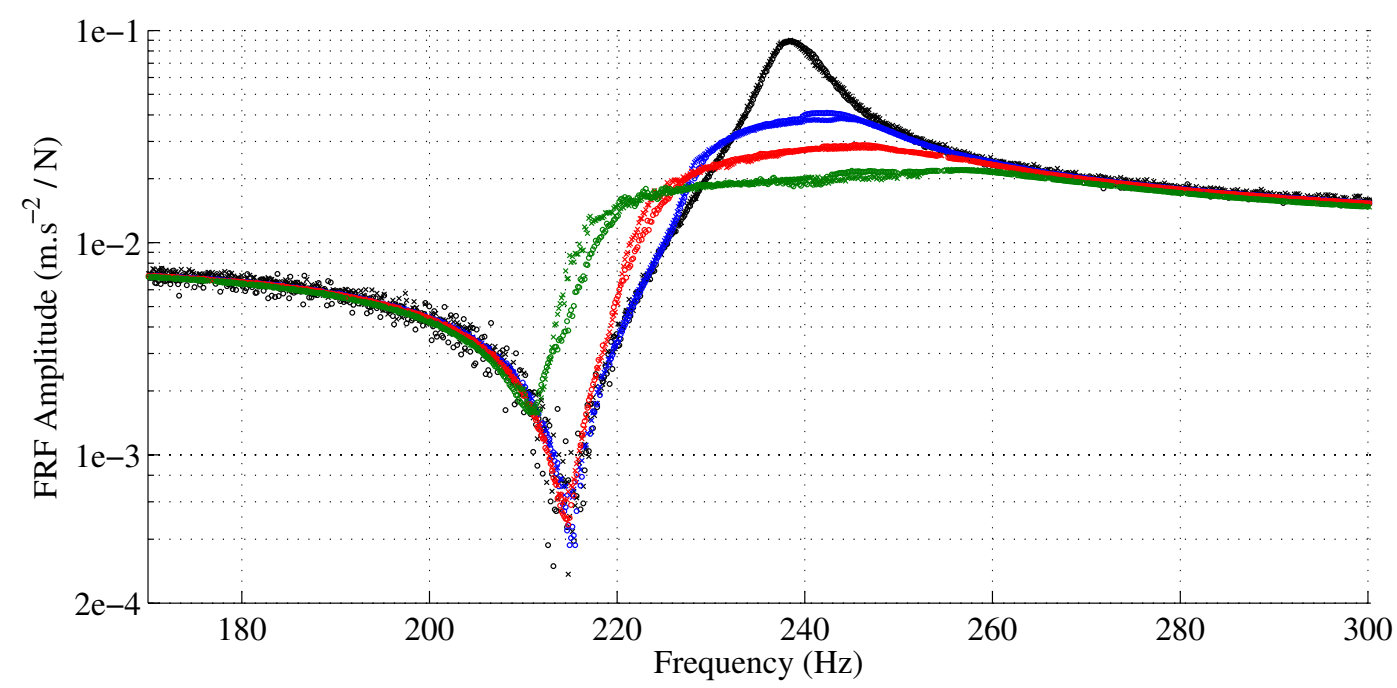

Figure 7: Experimental FRF on the base plate with increasing excitation level. The color legend is the same that in Fig. 6.

\begin{tabular}{|l|l|l|l|}
\hline Substructure & Mode & $\begin{array}{l}\text { Numerical resonance } \\
\text { frequency }\end{array}$ & $\begin{array}{l}\text { Error with } \\
\text { experiments }\end{array}$ \\
\hline $\begin{array}{l}\text { Substructure 1: } \\
\text { Envelope }\end{array}$ & $1^{\text {st }}$ vertical mode & $880.4 \mathrm{~Hz}$ & $1 \%$ \\
\hline $\begin{array}{l}\text { Substructure 2: } \\
\text { Central Body + Blades }\end{array}$ & $\begin{array}{l}1^{\text {st }} \text { vertical mode (blades and } \\
\text { central body synchronous) }\end{array}$ & $210.8 \mathrm{~Hz}$ & $3.6 \%$ \\
\hline $\begin{array}{l}\text { Substructure 2: } \\
\text { Central Body + Blades }\end{array}$ & $\begin{array}{l}4 \text { modes involving blades } \\
\text { asynchronously }\end{array}$ & {$[254.0-260.0] \mathrm{Hz}$} & $\simeq 3 \%$ \\
\hline
\end{tabular}

Table 4: Substructure resonance frequencies of the numerical model and comparison with tests.

experimental results (Sect. 2.3). For the second substructure, in the range [254-260]Hz, there are 4 modes involving blades that are due to the fact that there are 4 blades mounted on the central body with an axial symmetry. These 4 modes can be correlated to the several frequency peaks experimentally observed in a narrow frequency range (see Section 2.3). The eigenfrequencies obtained are compared with experimental results in Table 4.

Then the assembly model is validated with experiments at low excitation level. At low excitation level, friction is not initiated and the joints behave linearly. The numerical resonance frequencies are compared with tests in Table 5 for the two first resonances of the structure. The deformed shapes are plotted in Fig. 11. A deformed shape correlation analysis is performed, taking into account the $3 \times 10$ experimental acceleration signals. The results are presented in Table 5. Shape correlation calculations 


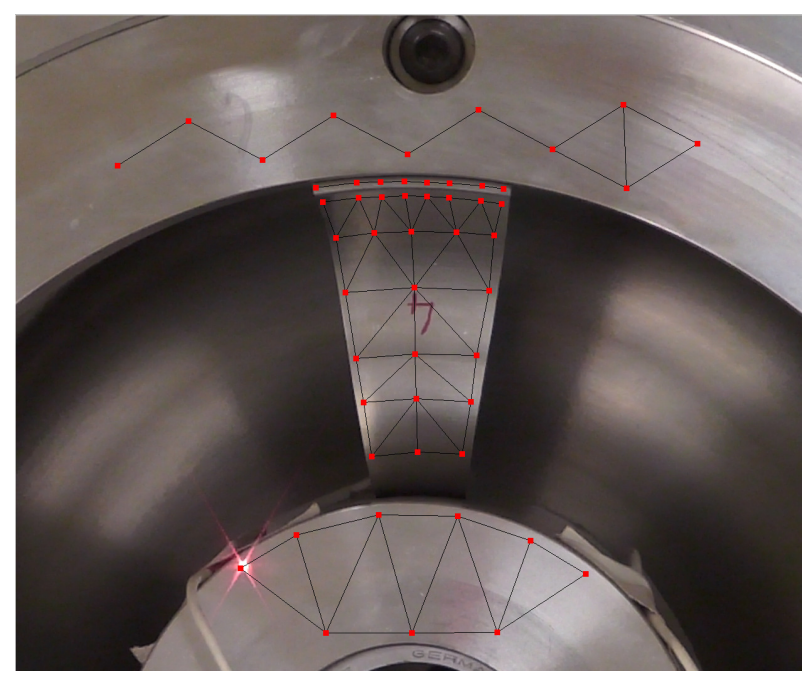

Figure 8: Location of the measurement points for the laser vibrometry.

\begin{tabular}{|l|l|l|l|}
\hline Mode & $\begin{array}{l}\text { Numerical resonance } \\
\text { frequency }\end{array}$ & $\begin{array}{l}\text { Error with } \\
\text { experiments }\end{array}$ & $\begin{array}{l}\text { Shape correlation } \\
\text { DAC }\end{array}$ \\
\hline $1^{\text {st }}$ vertical mode & $237.6 \mathrm{~Hz}$ & $0.1 \%$ & 99.8 \\
\hline $2^{\text {nd }}$ vertical mode & $878.4 \mathrm{~Hz}$ & $0.6 \%$ & 88.9 \\
\hline
\end{tabular}

Table 5: Assembly resonance frequencies of the numerical model and comparison with tests.

are performed using the Displacement Assurance Criterion definition [27]:

$$
D A C=100 \frac{\left|T\left\{\Psi_{e x p}\right\}\left\{\Psi_{\text {fem }}\right\}\right|^{2}}{\left.\left|T\left\{\Psi_{\text {fem }}\right\}\left\{\Psi_{\text {fem }}\right\}\right|\right|^{T}\left\{\Psi_{\text {exp }}\right\}\left\{\Psi_{\text {exp }}\right\} \mid}
$$

where $\Psi_{\text {exp }}$ is the vector of experimental complex response amplitude, $\Psi_{\text {fem }}$ is the vector of the complex response amplitude calculated from the finite element model, the complex conjugate transposed vector is denoted ${ }^{T}$.

A modal shape correlation was not possible because of the pilot signal. The complex force input signal that should be used for an experimental modal analysis was not available. In the narrow frequency range considered, the amplitude of the pilot tension is assumed to be proportional to the amplitude of the force. This assumption is wrong for the phase of the input signal, whereas the input signal phase is necessary for modal analysis. The force-tension equivalence assumption is tested in Fig. 12. Experimental data, plotted with crosses, is the measured response to a constant excitation tension amplitude of $20 \mathrm{mV}$. Three measurement points are plotted: top of the body (in blue), top edge of the envelope (in green) and center of the base plate (in red). The simulation results in the same points are plotted with plain curves. These results are obtained with a constant excitation force amplitude of $70 \mathrm{~N}$. Simulation and experimental resonance and anti-resonance frequencies match with less than $0.1 \%$ errors. The correlation is very good in the vicinity of the resonance but the asymptotic behaviors at $170 \mathrm{~Hz}$ and $300 \mathrm{~Hz}$ are different, revealing the limits of the assumption. 

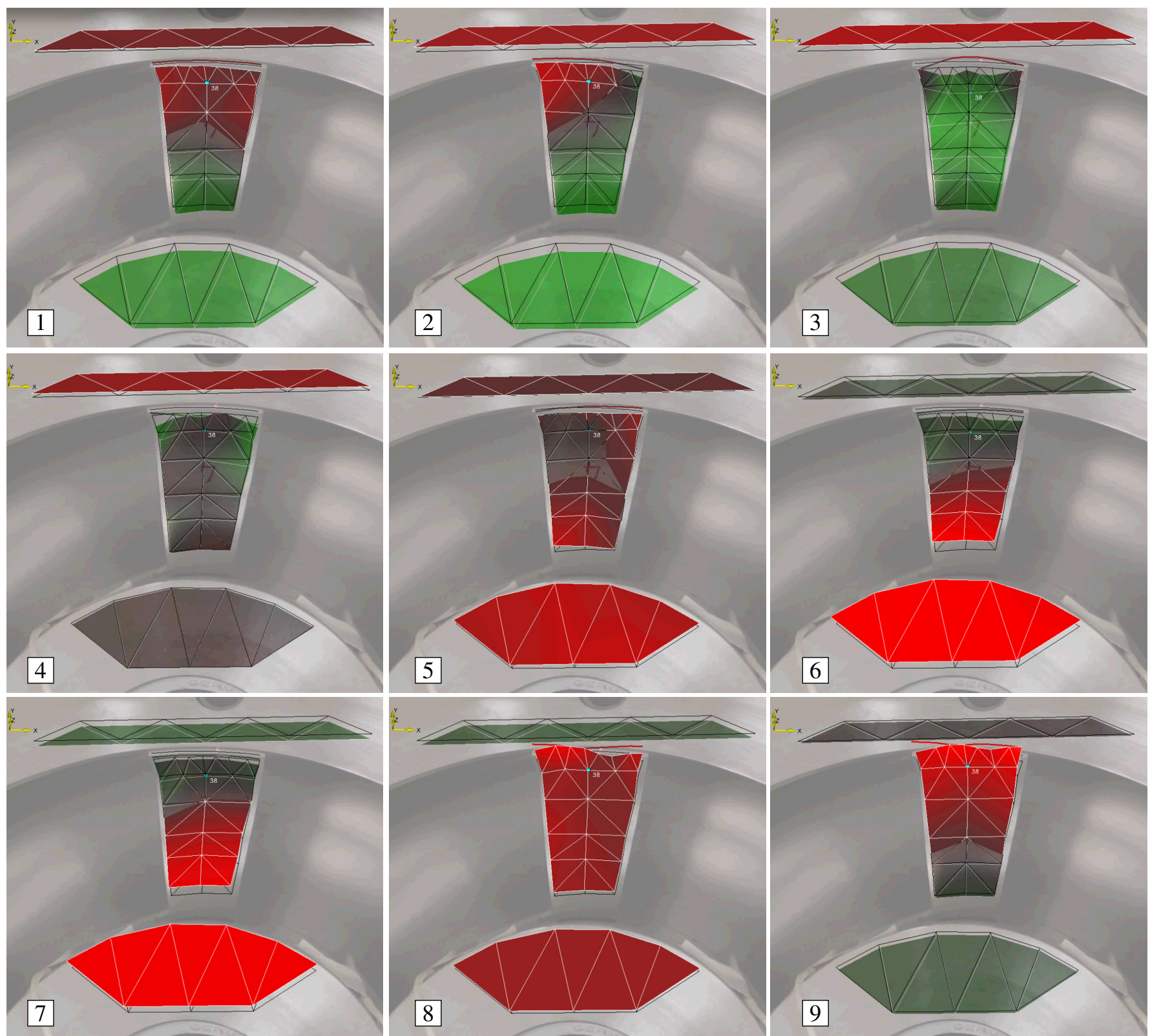

Figure 9: Deformed shape evolution of the blade during friction. Measurements obtained using laser vibrometry with a fixed sinusoidal excitation $(238 \mathrm{~Hz}, 3420 \mathrm{~N})$. 

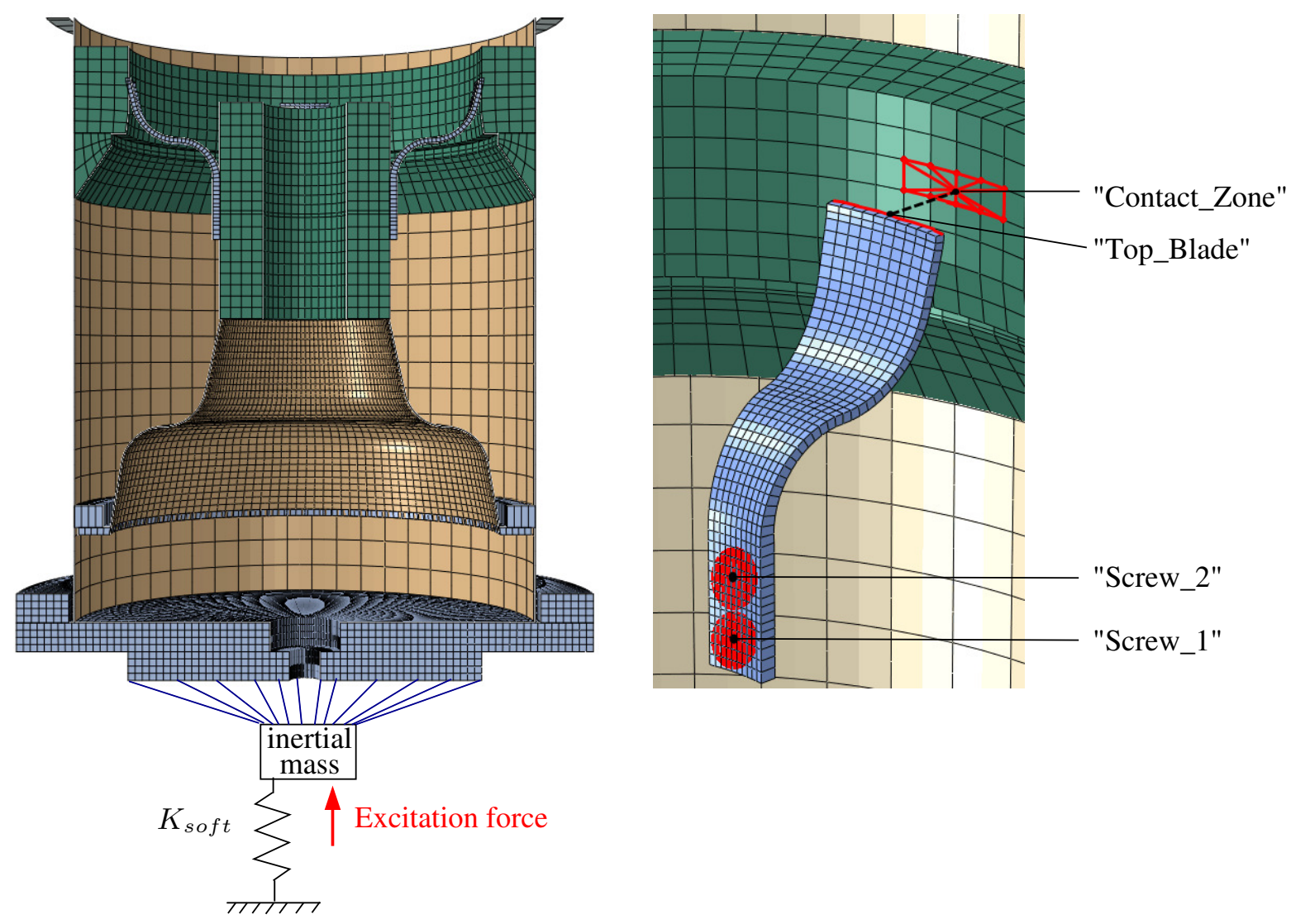

Figure 10: Cross-section view of the finite element model (left) and focus on the contact zone (right)

\subsection{Model reduction}

Nonlinear simulations are computationally expensive, which is incompatible with a detailed finite element model. The model presented in the previous section includes 278,848 degrees of freedom (DOFs). In practical terms the calculation results are of interest on only a few physical DOFs but the dynamic behavior of the full system is needed. The substructuring methods serve to reduce the size of a substructure model, keeping only the boundary DOFs and a few internal DOFs to keep the good dynamic behavior. Substructuring is used here as a reduction method; only one substructure is considered and the retained DOFs are not necessarily situated at the boundaries of the structure. The "Harmony" structure is considered as one substructure. The DOFs of the nodes corresponding to the 10 accelerometers' locations are retained. The vertical displacement of the inertial mass is also retained to apply the excitation force. For each blade, 3 nodes are retained to be able to introduce a nonlinear joint model between them. The 2 reference nodes driving the screw influence zones on the blade and the one driving the contact zone on the envelope are retained (see Fig. 10). Note that the reference node driving the top of the blade, where slipping actually occurs, is voluntarily not retained. The explanation is given in Section 4.4.

The most commonly used substructuring method is the one proposed by Craig and Bampton [28]. 

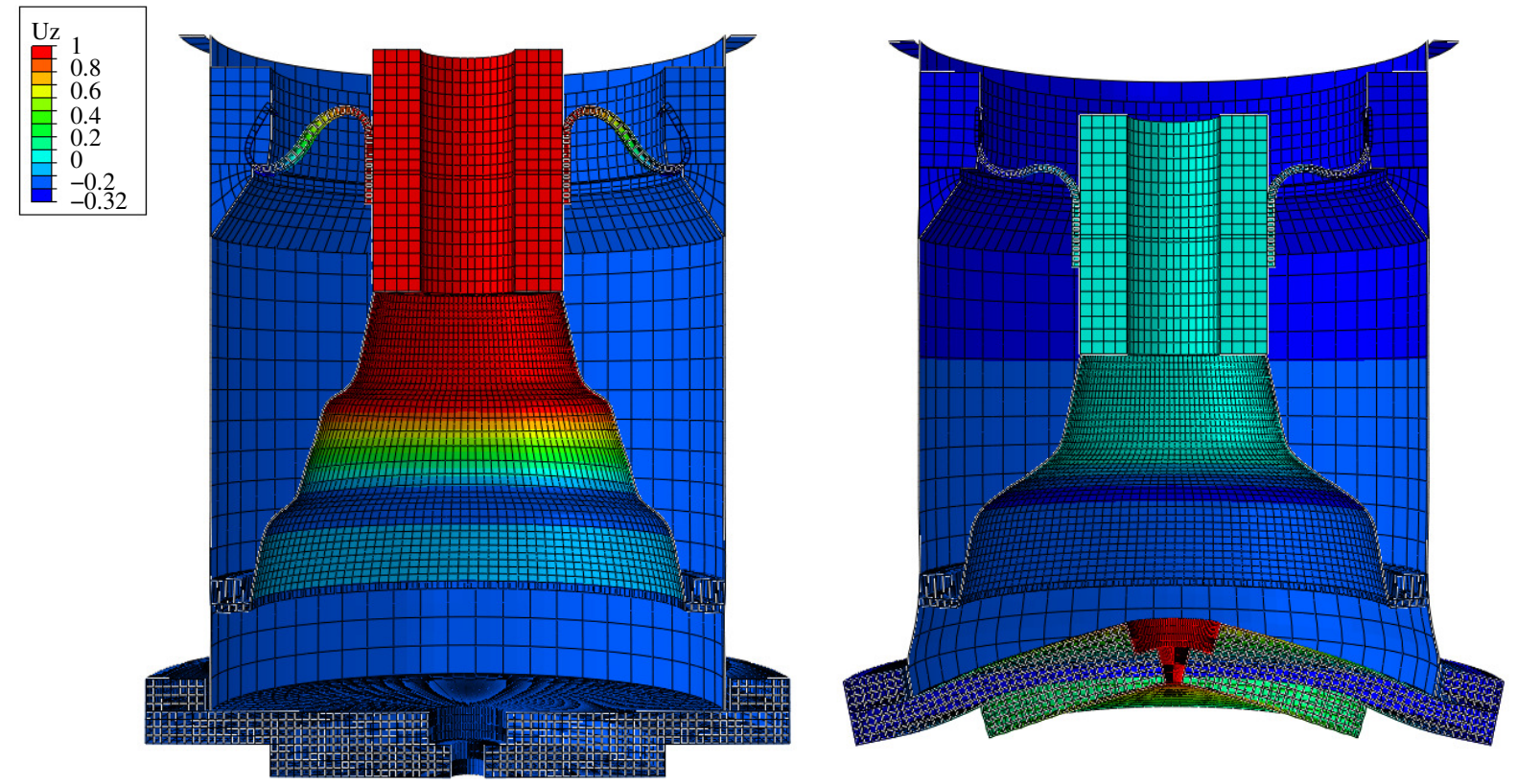

Figure 11: Cross-section view of the first (left) and second (right) axial mode (237.6Hz and $878.4 \mathrm{~Hz})$. The color represents the vertical displacement.

The reduction vectors are the static modes and the eigenmodes calculated with the retained DOFs blocked. The static modes, also called constraint modes, are the static response of the structure to a unit displacement imposed on one DOF and all the others blocked. The eliminated and retained DOFs are denoted respectively $e$ and $r$. The static modes are denoted $\Psi_{r}$ and satisfy the relations:

$$
\boldsymbol{\Psi}_{r}=\left\{\begin{array}{c}
\left.\boldsymbol{\Psi}_{r}\right|_{e} \\
\mathbf{1}_{r}
\end{array}\right\} \quad \text { and } \quad \mathbf{K}_{\mathrm{full}} \cdot \mathbf{\Psi}_{r}=\left[\begin{array}{cc}
\mathbf{K}_{\mathrm{ee}} & \mathbf{K}_{\mathrm{er}} \\
{ }^{T} \mathbf{K}_{\mathrm{er}} & \mathbf{K}_{\mathbf{r r}}
\end{array}\right]\left\{\begin{array}{c}
\left.\boldsymbol{\Psi}_{r}\right|_{e} \\
\mathbf{1}_{r}
\end{array}\right\}=\left\{\begin{array}{l}
\mathbf{0} \\
\mathbf{F}_{\mathbf{r}}
\end{array}\right\}
$$

SO

$$
\boldsymbol{\Psi}_{r}=\left\{\begin{array}{c}
-\mathbf{K}_{\mathbf{e e}}{ }^{-1} \mathbf{K}_{\mathrm{er}} \mathbf{1}_{r} \\
\mathbf{1}_{r}
\end{array}\right\}
$$

where $\mathbf{1}_{r}={ }^{T}[0, \ldots, 0,1,0, \ldots, 0]$ with 1 in the $r^{\text {th }}$ position and $\mathbf{K}_{\text {full }}$ is the stiffness matrix of the full model.

The substructuring method employed is a variant of the Craig-Bampton method. The eigenmodes $\mathbf{\Phi}_{\alpha}$ are calculated with no additional boundary conditions on the retained DOFs. As only one substructure is considered, these eigenmodes are exactly the ones of the structure studied. The method is specific to Abaqus code and is patented [29]. In the code, the family of vectors consisting of the static vectors $\boldsymbol{\Psi}_{r}$ and the eigenvectors $\boldsymbol{\Phi}_{\alpha}$ are modified through linear combinations to become a reduction basis compatible with the Craig-Bampton method. The static vectors are not modified. The values of eigenvectors at the retained DOFs are canceled using the static modes:

$$
\boldsymbol{\Phi}_{\alpha} \rightarrow \boldsymbol{\Phi}_{\alpha}^{*}=\boldsymbol{\Phi}_{\alpha}-\sum_{r} \Phi_{\alpha, r} \boldsymbol{\Psi}_{r}=\left[\begin{array}{c}
\boldsymbol{\Phi}_{\alpha, e}^{*} \\
\mathbf{0}_{r}
\end{array}\right]
$$




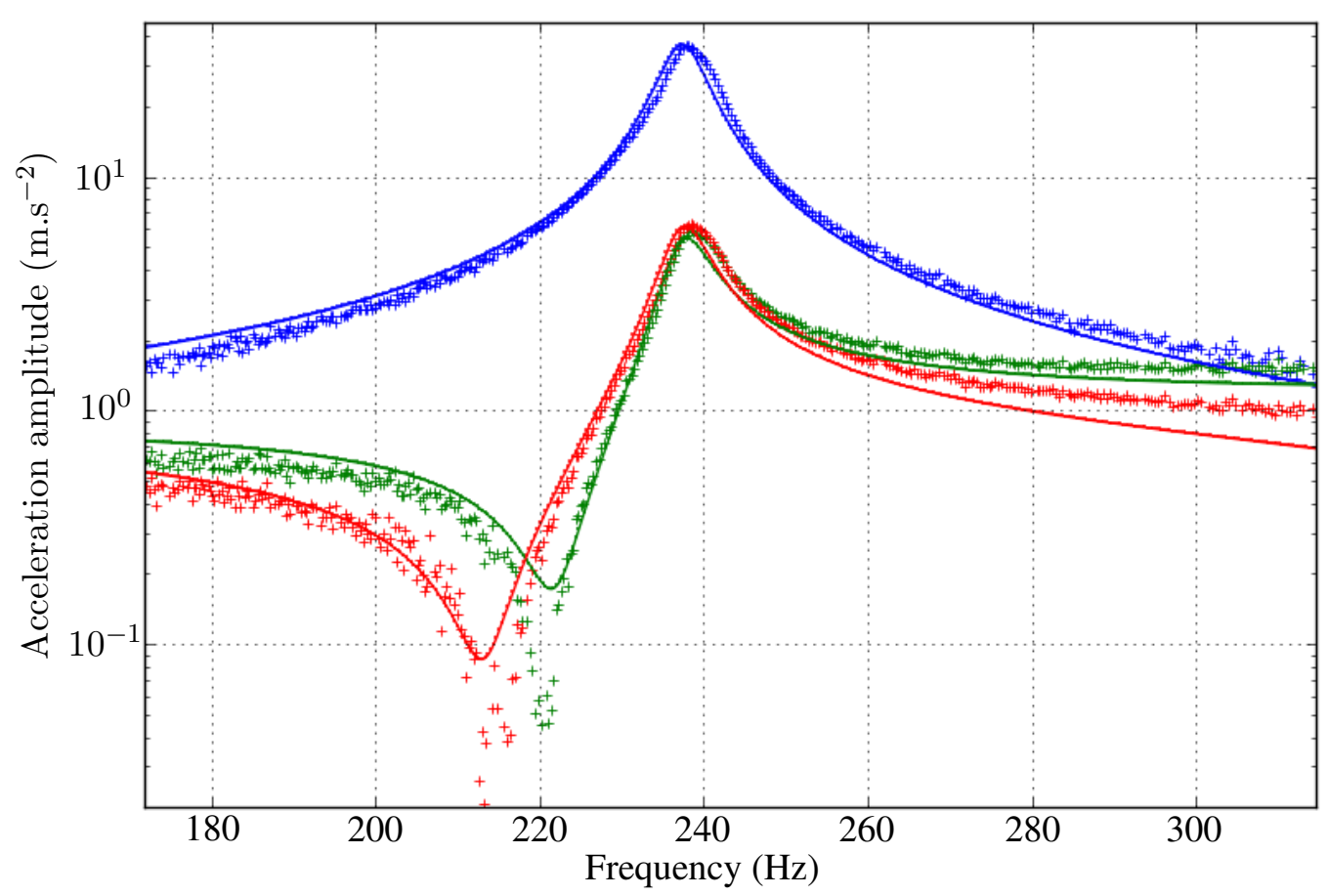

Figure 12: Comparison between experimental (crosses) and finite element simulation (plain curves) results on three distinct experimental points for a low excitation level : top of the body (blue), top edge of the envelope (green) and center of the base plate (red).

The obtained family of vectors is orthogonalized with respect to the inner product $\langle\mathbf{a} \mid \mathbf{b}\rangle={ }^{\mathbf{T}} \mathbf{a} \mathbf{K} \mathbf{b}$ and becomes the reduction basis. This basis has the same properties as a Craig-Bampton obtained basis:

- The static modes are unitary on 1 retained DOF and null on the others.

- The modified eigenmodes are null on the retained DOFs.

- The basis is orthogonal with respect to the inner product $\langle\mathbf{a} \mid \mathbf{b}\rangle={ }^{\mathbf{T}} \mathbf{a} \mathbf{K} \mathbf{b}$

The only difference with Craig-Bampton is that the retained eigenmodes of the reduced model are exactly those of the full model. For the "Harmony" structure, 103 nodal DOFs are retained and 34 eigenvectors are added. The linear dynamic behavior of the full and the reduced models are identical in the range [0-2000]Hz. The difference between the full and the reduced model's dynamical behavior appears with friction. The eigenvectors are calculated with the joints modeled by hard connectors, friction changes this condition and thus the modal basis. An alternative reduction method adapted to this kind of problem has been presented by Zucca and Epureanu [30]. The eigenvectors obtained with a free slipping condition in the joints are added to the reduction basis. As friction is a transition between stuck and slipping conditions in the joints, such addition will lower the precision losses due to reduction. This "advanced" reduction method has not been implemented for an industrial reason; the 


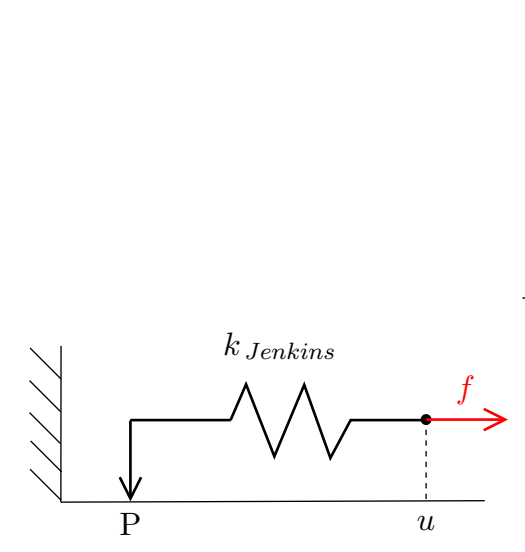

(a)

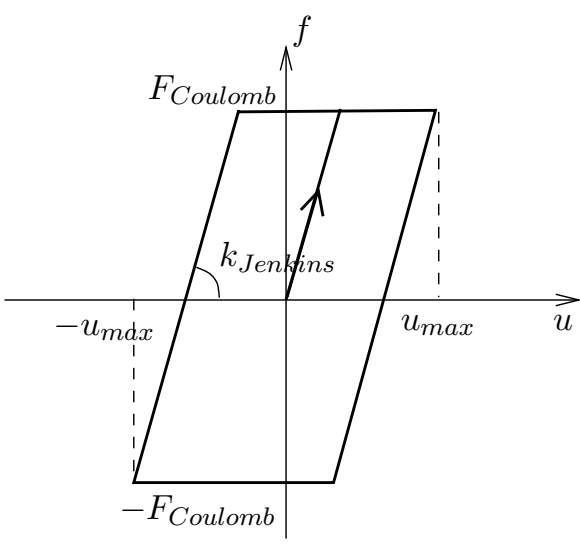

(b)

Figure 13: Schema (a) and force-displacement diagram (b) of a Jenkins friction model.

substructuring method presented is directly proposed by the industrial finite element code "Abaqus", it is thus easy to use from an engineering point of view, and yet precise enough. Anyway, no linear reduction method (such as the ones proposed by Craig and Bampton, by the software ABAQUS or by Zucca and Epureanu) provide an exact reduction of this nonlinear problem that include friction with such a few DOFs retained. All these linear reduction methods converge to the results given by the complete model if enough modes are considered.

Finally, the dynamical equation is written in the reduced basis:

$$
\mathbf{M} \ddot{\mathbf{U}}+\mathbf{D} \dot{\mathbf{U}}+\mathbf{K U}=\mathbf{F}_{\text {excit }} \cos (\Omega t)
$$

where $\mathbf{U}$ is the displacement in the reduced basis, $\mathbf{M}, \mathbf{D}$ and $\mathbf{K}$ are respectively the reduced mass, damping and stiffness matrices and $\mathbf{F}_{\text {excit }}$ is the projection of the excitation vector in the reduced basis (the excitation is along the vertical DOF of the inertial mass that is retained in the reduced basis). The damping matrix is defined according to a mass-proportional scheme : $\mathbf{D}=\alpha \mathbf{M} . \alpha$ has been updated using the low excitation level experiments in the vicinity of the first vertical mode.

\subsection{Friction modeling}

Different empirical/heuristic friction models have been proposed and developed to simulate the friction effects on mechanical systems and to predict restoring forces in mechanical contacts: the Coulomb friction model, the Dahl model [31], the Iwan model [32], the Valanis model [33] and the LuGre friction model [25] for example. In the present study, the numerical simulations are carried out with a 1-dimensional Masing friction model (also called Jenkins element, elastic Coulomb model or Prandtl model) for every blade. This model allows a better representation of the measured hysteresis than the Coulomb friction model. The idea of this well-known Masing model is to replace the blade stiffness by a Jenkins element, composed of a linear spring in series with a Coulomb slider (as illustrated in Fig. 13(a)). The slider remains in the stuck position until the force in the spring reaches the friction 
force when slipping begins. This force threshold $F_{\text {Coulomb }}$ is the unique parameter of the model.

The first step consists in defining the application vector of the Masing model in the reduced model. In the full finite element model, slipping is obtained by canceling the vertical component of the connector linking the points "Top_Blade" and "Contact_Zone" (see Fig. 13(b)). This component is defined as the application vector of the friction model in the complete model. The application vector of friction in the reduced model $\mathbf{V}_{\text {Jenkins }}$ is obtained by a projection in the reduced basis. $\mathbf{V}_{\text {Jenkins }}$ is normalized. The associated stiffness is denoted $k_{\text {Jenkins }}$, and the associated displacement is denoted $u$.

$$
\begin{aligned}
k_{\text {Jenkins }} & ={ }^{T} \mathbf{V}_{\text {Jenkins }} \mathbf{K} \mathbf{V}_{\text {Jenkins }} \\
u & ={ }^{T} \mathbf{V}_{\text {Jenkins }} \mathbf{U}
\end{aligned}
$$

where ${ }^{T}$ defines the transposed vector.

The frictional force is calculated using the iterative formulation introduced by Guillen and Pierre [34]. For each time iteration $i$, in a first step, a trial force is estimated assuming that the friction slider remains in the stuck position between the iteration $i-1$ and $i$ :

$$
{ }^{t r} f=f_{i-1}+k_{\text {Jenkins }}\left(u_{i}-u_{i-1}\right)
$$

Then the friction threshold $F_{\text {Coulomb }}$ is applied to calculate the force in the Jenkins element at the iteration i. This threshold creates 2 states; slip and stick. When the slip threshold is reached, we assumed a classical friction behavior. In the reverse case, no energy is dissipated and the contact behavior is purely elastic, as illustrated in Fig. 13(b).

$$
f_{i}=\left\{\begin{array}{lll}
{ }^{t r} f & \text { if }\left|{ }^{t r} f\right|<F_{\text {Coulomb }} & \text { stick } \\
\operatorname{sgn}\left({ }^{t r} f\right) F_{\text {Coulomb }} & \text { if }\left|{ }^{t r} f\right| \geq F_{\text {Coulomb }} & \text { slip }
\end{array}\right.
$$

Once applied to a periodic motion, the force-displacement diagram depicted in Fig. 13(b) is obtained.

As the Jenkins model replaces the linear model of the blade, the nonlinear force at iteration $i$ is defined as:

$$
\mathbf{F}_{\mathbf{n l}}\left(u_{i}\right)=\left(k_{\text {Jenkins }} \cdot u_{i}-f_{i}\right) \mathbf{V}_{\text {Jenkins }}
$$

The dynamical equation (6) become:

$$
\mathbf{M} \ddot{\mathbf{U}}+\mathbf{D} \dot{\mathbf{U}}+\mathbf{K U}=\mathbf{F}_{\text {excit }} \cdot \cos (\Omega t)+\mathbf{F}_{\mathbf{n l}}(\mathbf{U})
$$

\section{Nonlinear simulation}

As seen in the previous section, the frictional model is nonlinear and requires specific methods to compute nonlinear responses to forced excitation. In the present study, the stationary solution of the non-linear equation (12) is sought. In a previous work, the authors compared different nonlinear vibration simulation methods [7]. The well-known Harmonic Balance Method appears to be an efficient numerical method in order to calculate directly the system response on several harmonics. This method is coupled to a continuation method to browse the entire frequency range. As nonlinear calculations are computationally expensive, a condensation method is introduced to reduce the size of the nonlinear 
problem to its minimum. It is worth noting that the assumed steady-state periodic character of the vibrational response may be restrictive in context of mechanical systems with frictional junctions. Sometimes, non-periodic steady-state vibrations are reported which cannot be addressed and simulated by the use of the Harmonic Balance Method. In fact, the choice of estimating a stationary periodic solution can be seen as a limitation for comparisons between the numerical simulations and experiments in the present study.

\subsection{Harmonic Balance Method}

In this subsection, the methodology and use of the well-known Harmonic Balance Method [35] is briefly presented in the case of the present study.

As the excitation term is periodic, it is assumed that the nonlinear dynamical response and the force vector may be approximated by finite Fourier series with $\Omega$ as fundamental frequency.

$$
\begin{gathered}
\mathbf{U}(t)=\mathbf{B}_{0}+\sum_{k=1}^{m}\left(\mathbf{B}_{k} \cos (k \Omega t)+\mathbf{A}_{k} \sin (k \Omega t)\right) \\
\mathbf{F}_{\mathbf{n l}}(t)=\mathbf{C}_{0}+\sum_{k=1}^{m}\left(\mathbf{C}_{k} \cos (k \Omega t)+\mathbf{S}_{k} \sin (k \Omega t)\right)
\end{gathered}
$$

$\mathrm{m}$, the order of the Fourier series is selected on the basis of the number of significant harmonics expected in the nonlinear dynamical response. For our application, $\mathrm{N}=7$ has been chosen. Then, considering the fact that $\mathbf{F}_{\text {excit }}(t)=\mathbf{C}_{1 \text {, excit }} \cos (\Omega t)$, the equation (12) is rewritten in the Fourier basis:

$$
\begin{aligned}
& \mathbf{K B}_{0}=\mathbf{C}_{0} \\
& {\left[\begin{array}{cc}
\mathbf{K}-\Omega^{2} \mathbf{I}_{d} & -\Omega \mathbf{D} \\
\Omega \mathbf{D} & \mathbf{K}-\Omega^{2} \mathbf{I}_{d}
\end{array}\right]\left[\begin{array}{l}
\mathbf{A}_{1} \\
\mathbf{B}_{1}
\end{array}\right]=\left[\begin{array}{l}
\mathbf{S}_{1} \\
\mathbf{C}_{1}
\end{array}\right]+\left[\begin{array}{c}
0 \\
\mathbf{C}_{1, \text { excit }}
\end{array}\right]} \\
& {\left[\begin{array}{cc}
\mathbf{K}-(k \Omega)^{2} \mathbf{I}_{d} & -k \Omega \mathbf{D} \\
k \Omega \mathbf{D} & \mathbf{K}-(k \Omega)^{2} \mathbf{I}_{d}
\end{array}\right]\left[\begin{array}{l}
\mathbf{A}_{k} \\
\mathbf{B}_{k}
\end{array}\right]=\left[\begin{array}{c}
\mathbf{S}_{k} \\
\mathbf{C}_{k}
\end{array}\right] \quad \forall k \in[2, N]}
\end{aligned}
$$

where $\mathbf{I}_{d}$ is the identity matrix.

The coefficients $\mathbf{C}_{0}, \mathbf{S}_{k}$ and $\mathbf{C}_{k}$ are calculated by applying the Alternating Frequency Time domain method (AFT-method) that has been presented by Cameron and Griffin [36]. This method is summed up by the following scheme, where DFT means Discrete Fourier Transform:

$$
\mathbf{X}=\left[\mathbf{B}_{0} \mathbf{A}_{1} \mathbf{B}_{1} \ldots \mathbf{A}_{N} \mathbf{B}_{N}\right] \stackrel{D F T^{-1}}{\rightarrow} \mathbf{U} \rightarrow \mathbf{F}(\mathbf{U}) \stackrel{D F T}{\rightarrow}\left[\mathbf{C}_{0} \mathbf{S}_{1} \mathbf{C}_{1} \ldots \mathbf{S}_{N} \mathbf{C}_{N}\right]
$$

The Discrete Fourier Transform has been performed using 300 time steps over an excitation period. Finally, the discrete dynamical equation in the Fourier Basis can be rewritten:

$$
\mathbf{A} \mathbf{X}=\mathbf{W}_{\text {excit }}+\mathbf{W}_{\mathbf{n l}}(\mathbf{X})
$$

with 


$$
\begin{gathered}
\mathbf{A}=\operatorname{Diag}\left(\mathbf{K},\left\{\left[\begin{array}{cc}
\mathbf{K}-(k \Omega)^{2} \mathbf{I}_{d} & -k \Omega \mathbf{D} \\
k \Omega \mathbf{D} & \mathbf{K}-(k \Omega)^{2} \mathbf{I}_{d}
\end{array}\right]\right\}_{k=1, N}\right) \\
\mathbf{W}_{\text {excit }}=\left[\mathbf{0} \mathbf{0} \mathbf{C}_{1, \text { excit }} \mathbf{0} \ldots \mathbf{0}\right]_{2 N+1} \\
\mathbf{W}_{\mathbf{n l}}(\mathbf{X})=\left[\mathbf{C}_{0} \mathbf{S}_{1} \mathbf{C}_{1} \ldots \mathbf{S}_{N} \mathbf{C}_{N}\right]_{2 N+1}
\end{gathered}
$$

\subsection{Condensation procedure}

The computation time depends on the size of the nonlinear problem (16). A condensation procedure similar to the one proposed by Sinou [35], is introduced to reduce the size of the nonlinear problem. As the nonlinearity is local (in the contact region), many DOFs are not affected by $\mathbf{F}_{\mathbf{n l}}(\mathbf{U})$ and are thus related to the other DOFs through linear relations. The condensation procedure takes advantage of this localization of the nonlinearity to reduce the size of the problem to the number of DOFs actually affected by the nonlinear force. A basis change is necessary. First, the nonlinear DOFs are identified. The friction model introduced in Section 3.4 is a 1-dimension model with an application vector. As there are 4 blades, there are 4 independent nonlinear models. Each model is associated with a normalized application vector $\mathbf{V}_{i}$. In order to be more general, the number of nonlinear application vectors is denoted $\mathrm{q}$ (here $\mathrm{q}=4$ ). The q vectors $\left\{\mathbf{V}_{i}\right\}_{i=1 . . \mathrm{q}}$ are chosen to be the first vectors of the new basis. The new basis is obtained by applying the Gram-Schmit orthonormalization algorithm to the family of vectors $\left\{\mathbf{V}_{i}\right\}_{i=1 . . n+q}=\left\{\left\{\mathbf{V}_{i}\right\}_{i=1 . . q},\left[\mathbf{I}_{\mathbf{d}}\right]_{n}\right\}$ :

$$
\widetilde{\mathbf{V}}_{i}=\left\{\begin{array}{ll}
\frac{\widehat{\mathbf{V}}_{i}}{\left\|\widehat{\mathbf{V}}_{i}\right\|} & \text { if } \widehat{\mathbf{V}}_{i} \neq 0 \\
0 & \text { otherwise }
\end{array} \text { with } \widehat{\mathbf{V}}_{i}=\mathbf{V}_{i}-\sum_{k=1}^{i-1}\left(\mathbf{V}_{i} \mid \widetilde{\mathbf{V}}_{k}\right) \widetilde{\mathbf{V}}_{k}\right.
$$

At the end, the vectors that are null are eliminated and an orthonormal basis $\left\{\widetilde{\mathbf{V}}_{i}\right\}_{i=1 . . n}$ is obtained. In this new basis, only the DOFs associated with the first vectors $\left\{\widetilde{\mathbf{V}}_{i}\right\}_{i=1 . . q}$ are subjected to a nonlinear force. The application vectors $\left\{\mathbf{V}_{i}\right\}_{i=1 . . q}$ are expressed with only the $\left\{\tilde{\mathbf{V}}_{i}\right\}_{i=1 . . q}$ vectors:

$$
\mathbf{V}_{i}=\sum_{k=1}^{i-1}\left(\mathbf{V}_{i} \mid \widetilde{\mathbf{V}}_{k}\right) \widetilde{\mathbf{V}}_{k}+\left\|\mathbf{V}_{i}-\sum_{k=1}^{i-1}\left(\mathbf{V}_{i} \mid \widetilde{\mathbf{V}}_{k}\right) \widetilde{\mathbf{V}}_{k}\right\| \widetilde{\mathbf{V}}_{i} \quad \forall i=1 . . q
$$

The displacement vector $\mathbf{U}$ is expressed in the new basis, and separated between the q first nonlinear DOFs and the p next ones.

$$
\begin{gathered}
\left\{{ }^{T} \widetilde{\mathbf{V}}_{i}\right\}_{i=1 . . n} \mathbf{U}=\left[\begin{array}{c}
\mathbf{U}_{\mathbf{q}} \\
\mathbf{U}_{\mathbf{p}}
\end{array}\right] \\
{ }^{T} \widetilde{\mathbf{V}} \mathbf{M} \widetilde{\mathbf{V}}\left[\begin{array}{c}
\ddot{\mathbf{U}}_{\mathbf{q}} \\
\ddot{\mathbf{U}}_{\mathbf{p}}
\end{array}\right]+{ }^{T} \widetilde{\mathbf{V}} \mathbf{D} \widetilde{\mathbf{V}}\left[\begin{array}{c}
\dot{\mathbf{U}}_{\mathbf{q}} \\
\dot{\mathbf{U}}_{\mathbf{p}}
\end{array}\right]+{ }^{T} \widetilde{\mathbf{V}} \mathbf{K} \widetilde{\mathbf{V}}\left[\begin{array}{c}
\mathbf{U}_{\mathbf{q}} \\
\mathbf{U}_{\mathbf{p}}
\end{array}\right]={ }^{T} \widetilde{\mathbf{V}} \mathbf{F}_{\text {excit }}+\left[\begin{array}{c}
\mathbf{F}_{\mathbf{n l}}\left(\mathbf{U}_{\mathbf{q}}\right) \\
0
\end{array}\right]
\end{gathered}
$$

The same separation is done in the Fourier domain.

$$
\Lambda=\operatorname{Diag}\left(\tilde{\mathbf{V}}_{q}, \tilde{\mathbf{V}}_{q}, \ldots, \tilde{\mathbf{V}}_{q}, \tilde{\mathbf{V}}_{p}, \tilde{\mathbf{V}}_{p}, \ldots, \tilde{\mathbf{V}}_{p}\right)_{(2 N+1) n} \quad \text { with } \quad \tilde{\mathbf{V}}_{q}=\left\{\tilde{\mathbf{V}}_{i}\right\}_{i=1 . . q}, \quad \tilde{\mathbf{V}}_{p}=\left\{\tilde{\mathbf{V}}_{i}\right\}_{i=q . . n}
$$




$$
{ }^{T} \Lambda \mathbf{X}=\left[\begin{array}{l}
\mathbf{X}_{\mathbf{q}} \\
\mathbf{X}_{\mathbf{p}}
\end{array}\right] \quad{ }^{T} \Lambda \mathbf{W}_{\text {excit }}=\left[\begin{array}{l}
\mathbf{W}_{\text {excit }, \mathbf{q}} \\
\mathbf{W}_{\text {excit }, \mathbf{p}}
\end{array}\right] \quad{ }^{T} \Lambda \mathbf{A} \Lambda=\left[\begin{array}{cc}
\mathbf{A}_{\mathbf{q q}} & \mathbf{A}_{\mathbf{q p}} \\
\mathbf{A}_{\mathbf{p q}} & \mathbf{A}_{\mathbf{p p}}
\end{array}\right]
$$

The dynamical equation in the Fourier basis (16) becomes:

$$
\left[\begin{array}{ll}
\mathbf{A}_{\mathbf{q q}} & \mathbf{A}_{\mathbf{q p}} \\
\mathbf{A}_{\mathbf{p q}} & \mathbf{A}_{\mathbf{p p}}
\end{array}\right]\left[\begin{array}{l}
\mathbf{X}_{\mathbf{q}} \\
\mathbf{X}_{\mathbf{p}}
\end{array}\right]=\left[\begin{array}{l}
\mathbf{W}_{\text {excit }, \mathbf{q}} \\
\mathbf{W}_{\text {excit }, \mathbf{p}}
\end{array}\right]+\left[\begin{array}{c}
\mathbf{W}_{\mathbf{n l}}\left(\mathbf{X}_{\mathbf{q}}\right) \\
0
\end{array}\right]
$$

This expression serves to write a linear relation between $\mathbf{X}_{\mathbf{p}}$ and $\mathbf{X}_{\mathbf{q}}$ :

$$
\mathbf{X}_{\mathbf{p}}=\mathbf{A}_{\mathbf{p p}}{ }^{-1}\left(\mathbf{W}_{\text {excit }, \mathbf{p}}-\mathbf{A}_{\mathbf{p q}} \mathbf{X}_{\mathbf{q}}\right)
$$

Then Eq. (26) is expressed as an equation in $\mathbf{X}_{\mathbf{q}}$ :

$$
\left(\mathbf{A}_{\mathbf{q q}}-\mathbf{A}_{\mathbf{q p}} \mathbf{A}_{\mathbf{p p}}{ }^{-1} \mathbf{A}_{\mathbf{p q}}\right) \mathbf{X}_{\mathbf{q}}=\mathbf{W}_{\text {excit }, \mathbf{q}}-\mathbf{A}_{\mathbf{q p}} \mathbf{A}_{\mathbf{p p}}{ }^{-1} \mathbf{W}_{\text {excit }, \mathbf{p}}+\mathbf{W}_{\mathbf{n l}}\left(\mathbf{X}_{\mathbf{q}}\right)
$$

This last expression is solved in the same manner as Eq. (16). From equation (28), an optimization function $\mathbf{H}$ is defined:

$$
\mathbf{H}\left(\mathbf{X}_{\mathbf{q}}, \Omega\right)=\left(\mathbf{A}_{\mathbf{q q}}-\mathbf{A}_{\mathbf{q p}} \mathbf{A}_{\mathbf{p p}}{ }^{-1} \mathbf{A}_{\mathbf{p q}}\right) \mathbf{X}_{\mathbf{q}}-\mathbf{W}_{\text {excit }, \mathbf{q}}+\mathbf{A}_{\mathbf{q p}} \mathbf{A}_{\mathbf{p p}}{ }^{-1} \mathbf{W}_{\text {excit }, \mathbf{p}}-\mathbf{W}_{\mathbf{n l}}\left(\mathbf{X}_{\mathbf{q}}\right)
$$

The nonlinear dynamical equation has been transformed into the optimization problem:

$$
\mathbf{H}\left(\mathbf{X}_{\mathbf{q}}, \Omega\right)=\mathbf{0}
$$

The condensation procedure has reduced the size of the nonlinear problem from $(2 \mathrm{~N}+1) \mathrm{n}$ to $(2 \mathrm{~N}+1) \mathrm{q}$ DOFs. For this application, with $N=7$, the size is reduced from 2055 to 60 DOFs. The full solution $\mathbf{X}$ is then linearly computed from $\mathbf{X}_{\mathbf{q}}$ using Eqs. (27) and (25).

\subsection{Continuation procedure}

The problem (28) is solved using a continuation method, to browse the frequency range continuously. A predictor-corrector scheme has been implemented. The pseudo-arclength continuation method is chosen [6]. The arc length between two consecutive points $\left(\mathbf{X}_{\mathbf{q}}{ }^{(i)}, \Omega^{(i)}\right)$ and $\left(\mathbf{X}_{\mathbf{q}}{ }^{(i+1)}, \Omega^{(i+1)}\right)$ is defined as:

$$
\Delta s_{i+1}=\sqrt{\left\|\mathbf{X}_{\mathbf{q}}{ }^{(i+1)}-\mathbf{X}_{\mathbf{q}}{ }^{(i)}\right\|^{2}+\alpha\left|\Omega^{(i+1)}-\Omega^{(i)}\right|^{2}}
$$

$\alpha$ is a numerical coefficient introduced in order to take into account the difference of magnitude between $\Omega\left(\sim 10^{3} \mathrm{~s}^{-1}\right)$ and $\left\|\mathbf{X}_{\mathbf{q}}\right\|\left(\sim 10^{-4} \mathrm{~m}\right)$.

The continuation method is illustrated in Fig. 14. It starts with a prediction step. Considering that 3 points are already computed $\left(\mathbf{Y}_{i-2}, \mathbf{Y}_{i-1}\right.$ and $\mathbf{Y}_{i}$, where $\left.\mathbf{Y}_{i}=\left[\mathbf{X}_{\mathbf{q}}{ }^{(i)}, \Omega^{(i)}\right]\right)$, the next point is extrapolated. A second degree Lagrangian polynomial prediction is chosen. The calculation step $\Delta s$ between the prediction and the previous point is a fixed parameter of the algorithm. In case of convergence issues, an adaptive calculation step can be introduced.

Then comes the correction step. The Newton algorithm is used to move $\mathbf{Y}_{i+1}^{(0)}$ closer to a zero of $\mathbf{H}$. In the pseudo-arclength scheme, the direction of research $\mathbf{Y}_{i+1}-\mathbf{Y}_{i+1}^{(0)}$ is orthogonal to the direction of the prediction $\mathbf{Y}_{i+1}^{(0)}-\mathbf{Y}_{i}$, as illustrated in Fig. 14. Every correction step is given by the equation:

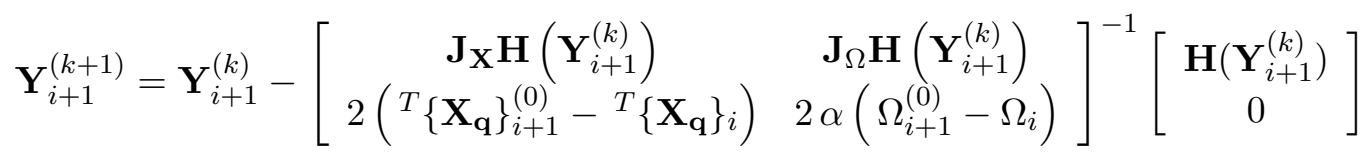




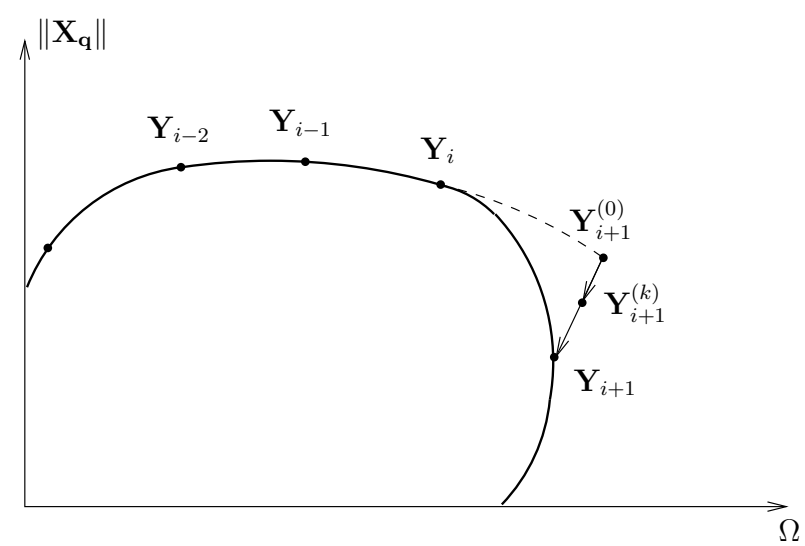

Figure 14: Schema of the continuation process.
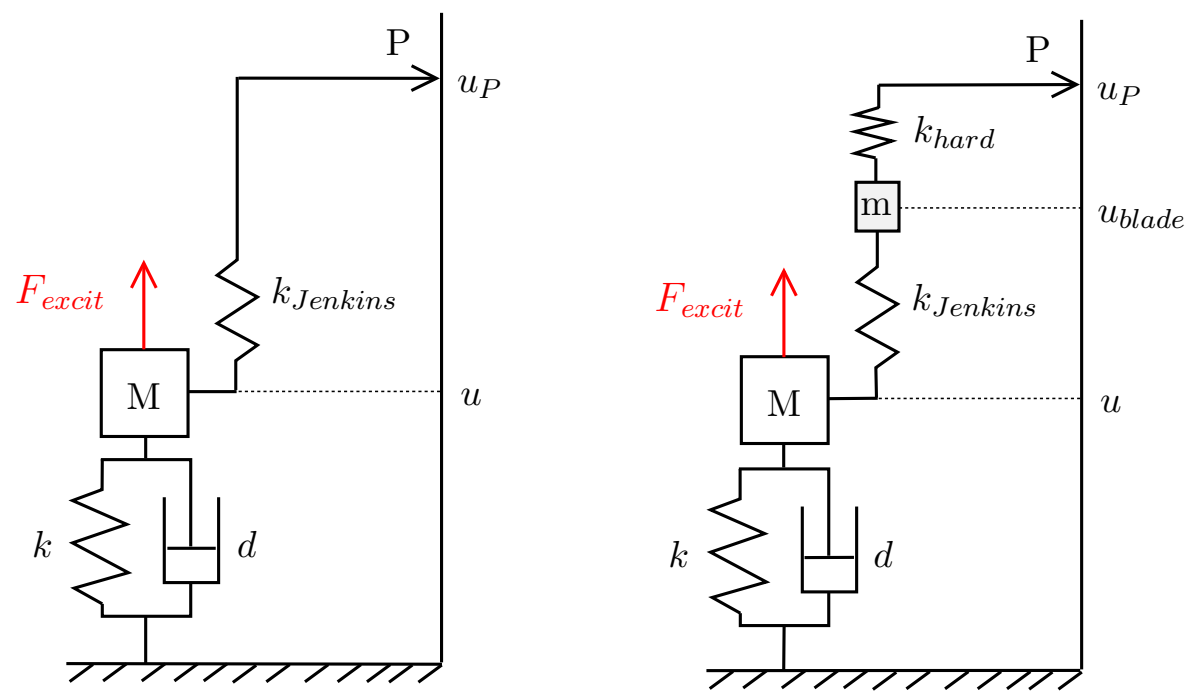

Figure 15: 1 degree of freedom friction system (left) and equivalent 2 degrees of freedom friction system (right)

The Newton iteration process is stopped when the numerical error is low enough: $\left|\mathbf{H}\left(\mathbf{Y}_{i+1}^{(k)}\right)\right|<\epsilon$ with $\epsilon$ the chosen numerical error level.

\subsection{Simulation of the blade movement}

A priori, the easiest way to calculate the movement of the top of the blade is to retain the corresponding DOF in the reduced model and then directly read its movement in the response vector. Doing this, a numerical convergence issue appears. In order to point out this issue and justify the modeling chosen, explanations are given on a very simple analogous system. In the vicinity of the first vertical mode, the "Harmony" system can be roughly modeled by the 1-DOF model presented in Fig. 15 (left). In 


\begin{tabular}{|l|l|}
\hline Parameter & Value \\
\hline$M$ & $10 \mathrm{~kg}$ \\
\hline$m$ & $0.001 \mathrm{~kg}$ \\
\hline$k$ & $2.2410^{7} \mathrm{~kg} \cdot \mathrm{s}^{-2}$ \\
\hline$k_{\text {Jenkins }}$ & $8.1110^{6} \mathrm{~kg} \cdot \mathrm{s}^{-2}$ \\
\hline$k_{\text {hard }}$ & $10^{9} \mathrm{~kg} \cdot \mathrm{s}^{-2}$ \\
\hline$d$ & $350 \mathrm{~kg} \cdot \mathrm{s}^{-1}$ \\
\hline$F_{\text {Coulomb }}$ & $1000 \mathrm{~N}$ \\
\hline$F_{\text {excit }}$ & $1000 \mathrm{~N}$ \\
\hline
\end{tabular}

Table 6: Parameters' values for the 1-DOF and 2-DOFs models.

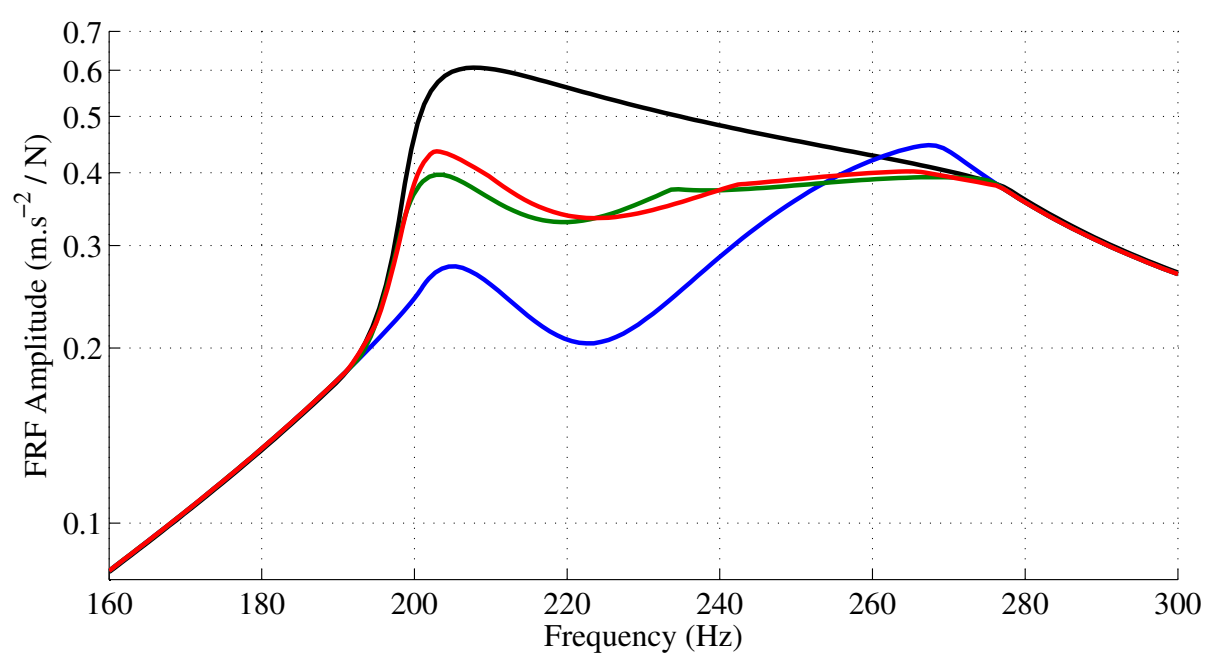

Figure 16: Simulated harmonic response of point A (see Fig. 15) in the 1-DOF system (black) and in the 2-DOFs system with different number $\mathrm{N}$ of harmonics taken into account in the HBM. $\mathrm{N}=1$ (blue) $\mathrm{N}=5$ (green) $\mathrm{N}=11$ (red).

this model, including the top of the blade as a retained DOF leads to the 2-DOFs model presented in Fig. 15 (right). The parameter values chosen for these models are given in Table 6 . The value of the little mass $\mathrm{m}$ introduced is chosen to have a negligible influence on the response of the system (i.e. the numerical results are the same with $\mathrm{m}$ divided by 10 or 100). With such a negligible additional mass, these two models should give exactly the same results on the DOF " $u$ ". Fig. 16 presents the results obtained with the 1 -DOF model and those obtained with the 2-DOFs model. The results obtained with the 1-DOF model -plotted in black- are not sensitive to $N$, the number of harmonics chosen in the Harmonic Balance Method. On the contrary, with the 2-DOFs model, the calculated FRF evolves a lot with the number of harmonics chosen. The results obtained with $N=1,5$ and 11 are plotted in Fig. 16. The results plotted are always the FRF deducted from the amplitude of harmonic 1 , the difference is that the calculations have been performed with various values of $N$ for the 


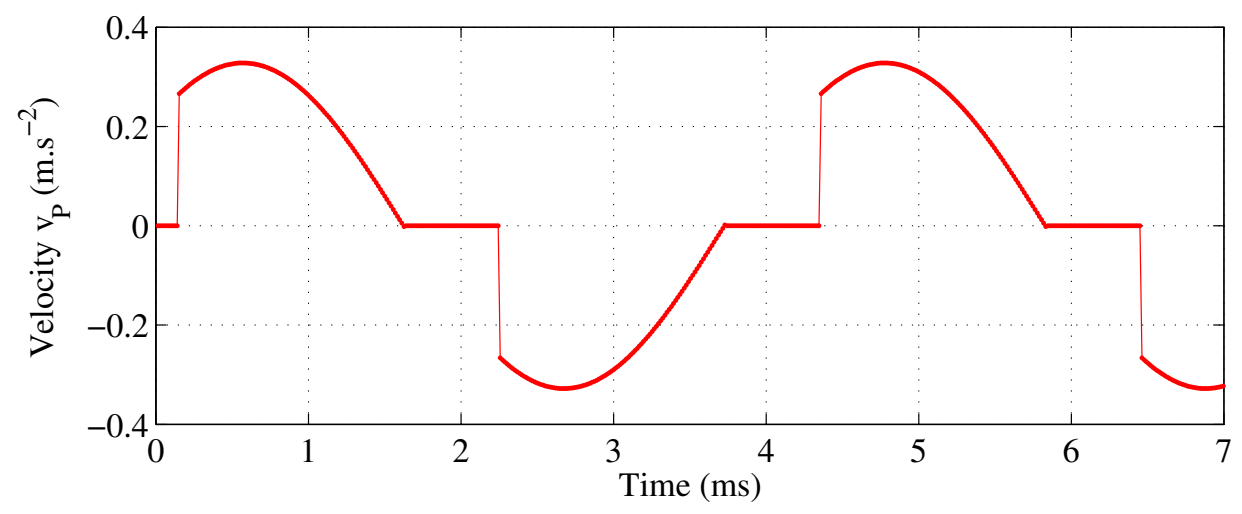

Figure 17: Time response of point $\mathrm{P}$ with the 1-DOF model. The response signal is calculated at $238 \mathrm{~Hz}$

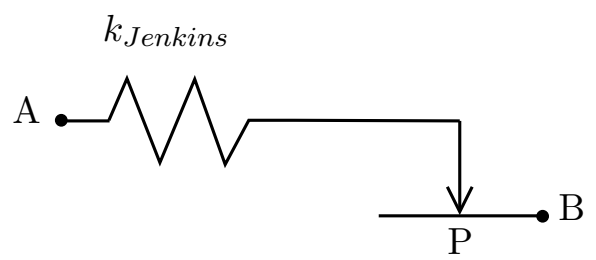

Figure 18: Schema of the blade model: a 2-nodes Jenkins element

Fourier truncation. The computation time appears to grow exponentially with $N$ and for $N>11$, the optimization algorithm fails to converge. In order to understand this issue, the velocity of the point $\mathrm{P}$ is computed. This computation is easy using the iterative formulation presented in Section 3.4:

$$
v_{P}(i)= \begin{cases}0 & \text { if stuck } \\ \dot{u}(i) & \text { if slipping }\end{cases}
$$

The velocity of point $\mathrm{P}$ in the 1-DOF model is depicted in Fig. 17. The velocity is discontinuous when slipping occurs. If a point very close to $\mathrm{P}$ is added to the model (Fig. 15, right), then this point and $\mathrm{P}$ have almost the same movement. In the Harmonic Balance Method, the displacements of all the DOFs are approximated by their truncated Fourier transform. The discontinuity revealed in Fig. 17, cannot be correctly approximated by a low order Fourier series. That is the reason why, even though the two models presented in Fig. 15 seem equivalent, their solutions using the Harmonic Balance Method, are strongly different. In the first case, the velocity of point $\mathrm{P}$ is free to be discontinuous whereas in the second case, it is constrained to behave as a truncated Fourier series. To avoid this issue, the number of harmonics can be increased but this directly leads to major convergence difficulties. The solution that has been chosen in this study is a workaround. The top of the blade is not retained in the reduced model. The convergence issue in the Harmonic Balance Method is thus avoided. The nonlinear calculations are performed with a 2-nodes blade model that is illustrated in Fig. 18. $v_{P}$ is calculated using Eq. (33). This velocity $v_{P}$ can then be directly compared with velocity measurements 
performed on the top of a blade using the scanning laser vibrometer. These comparisons are presented in Section 5.3. Alternative methods have been developed to overcome the problem presented in this section. Poudou [13, 37] has pointed out exactly the same issue, dealing with the computation of the frequency response of the 2-DOFs model presented in Fig. 15. He proposed to solve iteratively the problem with increasing values of $k_{\text {hard }}$. Another method developed by Nacivet et al. [14] is based on a Lagrangian formulation of the nonlinear force, coupled with a frequency-time method.

\section{Test-simulation comparison}

Experiments and simulations are compared at 3 different levels. The first comparison uses the FRF. Simulation reproduces the flattening and softening effect observed in experimental FRF (see Sect. 2.4). Then the appearance of multi-harmonic signals is studied. This onset is evidence of nonlinear vibration response that can be both measured and simulated. Last, the local movement of the blade during friction, measured using the scanning laser vibrometer (see Section 2.5), provides the opportunity to compare the predicted numerical results with experiments for the specific vibrational behavior near the contacts

\subsection{Frequency Response Functions}

The obtained simulated Frequency Response Functions are compared to experiments in Fig. 19 and 20. The Coulomb force is the unique updated parameter of the model, its updated value is $F_{\text {Coulomb }}=$ 290 N. Simulations reproduce the flattening of the resonance peak presented in Section 2.4 very well. The response of the structure is also simulated at higher excitation levels, where experiments are not available due to the threat of damaging the structure. Simulations at $6000 \mathrm{~N}$ and $10000 \mathrm{~N}$ excitation levels are plotted respectively in brown and violet dashed curves. An important softening effect is observed. The maximal resonance frequency is lowered. An asymptotic resonance at $190 \mathrm{~Hz}$ is observed. It corresponds to a mode where the blades slip freely on the envelope. Friction induces a transition from a resonant mode with the sliders in the stuck position to another with free slipping of the sliders. $\mathrm{Al}$ Sayed and al. [11] showed that the maximal flattening of the resonance peak is obtained when the percentage stick/slip over a period is around $50 \%$.

\subsection{Multi-harmonic comparison}

Beyond the study of the harmonic response presented in Section 5.1, a multi-harmonic comparison has been performed following the method previously published by the authors [7]. The different harmonics are directly calculated in the Harmonic Balance Method. The $1^{\text {st }}, 3^{r d}, 5^{\text {th }}$ and $7^{\text {th }}$ harmonics are plotted respectively in blue, green, red and black in Fig 21(a). Experimental harmonics are extracted using a signal processing tool [7] and plotted in Fig 21(b). The excitation level chosen for this multiharmonic comparison is $2910 \mathrm{~N}$. Friction occurs in the range [210-260]Hz. An increase of the $3^{r d}, 5^{\text {th }}$ and $7^{\text {th }}$ harmonic levels happens in this range. Experimental and simulated results are very similar. Simulation reproduces the harmonics generated by friction. In the $5^{\text {th }}$ harmonic simulated response, a resonance and an anti-resonance are observed at $238 \mathrm{~Hz}$ and $243 \mathrm{~Hz}$; these phenomena are not fully in agreement with measurements. This difference reveals the limits of the proposed Finite Element 


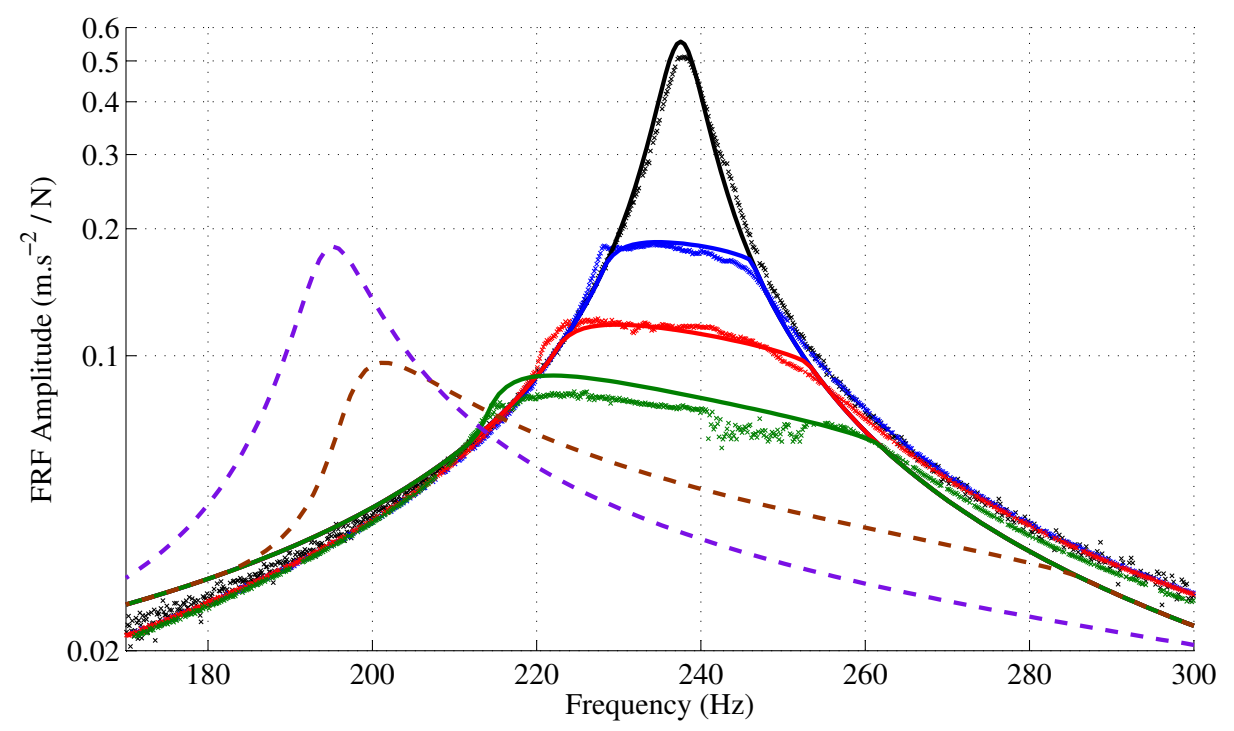

Figure 19: Comparison between experimental (crosses) and simulated (plain curves) FRF at the top of the central body for increasing excitation levels: $70 \mathrm{~N}$ (black), $1070 \mathrm{~N}$ (blue), $1875 \mathrm{~N}$ (red) and 2910N (green). Simulation results at higher excitation level are plotted with dashed curves: 6000N (brown) and $10000 \mathrm{~N}$ (violet).

Model in simulating the dynamic behavior of the real structure around $5 \times 238=1190 \mathrm{~Hz}$. Away from the frequency range where friction happens, the simulation model is linear so the $3^{r d}, 5^{\text {th }}$ and $7^{\text {th }}$ harmonics are not simulated. The experimental levels observed in this zone must be the harmonic components of the measurement noise, or can be explained by a non-ideal multi-harmonic excitation signal [7]. The accelerometer measurements are thus very well reproduced by simulation.

\subsection{Local stick-slip movement}

The use of the laser vibrometer presented in Section 2.5 enables a local study of the stick-slip movement. The mean vertical displacement of the top of the blade and the external envelope are plotted in Fig. 22 respectively in red and blue. In a stuck period, the two curves overlap. The separation of the two curves reveals slipping. The stick-slip movement is readable in Fig. 22. The movement of the top of the blade is then simulated using the workaround presented in Section 4.4. Fig. 23 presents these simulation results. The blade model used for this simulation is the 2-nodes Jenkins model illustrated in Fig. 18. The velocities of the slider (point P) and of the envelope (point B) in Fig. 23 are directly comparable with the measurements in Fig. 22. In Fig. 23, the discontinuous velocity of the sliding point $\mathrm{P}$ highlights the difficulty presented in Section 4.4. This sliding point $\mathrm{P}$ has a strongly nonharmonic and non-smooth movement that is very difficult to approximate with truncated Fourier series. Including such a point in the full nonlinear problem leads to strong convergence issues. The comparison with measurements shows that the simulated stick-slip movement of point $\mathrm{P}$ is in agreement with experiments. 


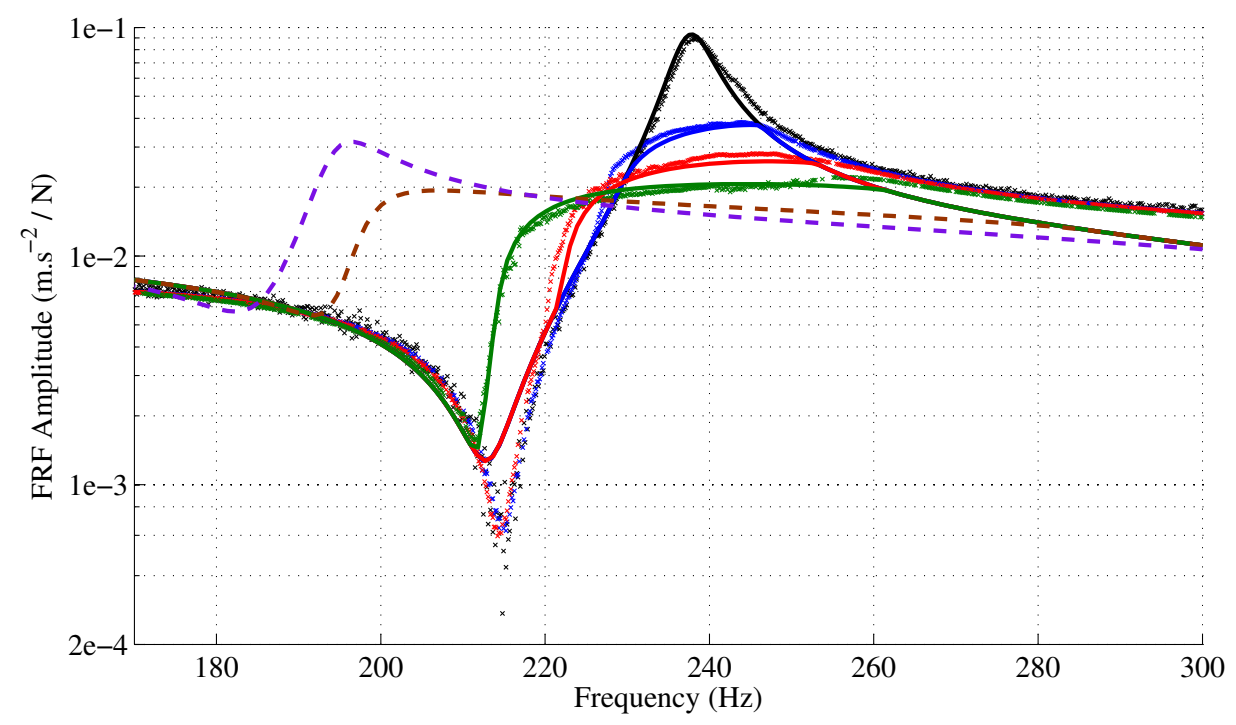

Figure 20: Comparison between experimental (crosses) and simulated (plain curves) FRF at the center of the base plate for increasing excitation levels. The color legend is the same as in Fig. 19.

\section{Conclusion}

A nonlinear vibration simulation method is successfully applied to a test structure with friction joints. The presented method is based on 4 main steps: finite element modeling, model reduction, nonlinear simulation and comparison with experimental results. The nonlinear simulation process uses the harmonic balance method coupled with continuation and condensation algorithms. It is observed that simulations reproduce the experimental flattening and shifting of the resonance peak when increasing the excitation level. The simulated multi-harmonic response due to friction is also in agreement with measurements. One of the original contributions of the present study is the use of a scanning laser vibrometer to measure the nonlinear behavior near the contacts. Thereby, comparison between experiments and predicted vibrations allows to increase the confidence in (or to understand the limitations of) the numerical models currently used for friction damping simulation. In our present study, the local analysis of the stick-slip movement in the friction zone using laser vibrometry shows that the simplified one-dimension and one-parameter friction model chosen (i.e. a macro character of the joint behavior) can be sufficient to reproduce the physical friction movement. This study demonstrates that the presented nonlinear simulation method is relevant in simulating the evolution of the frequency response due to friction in assemblies (frequency shifts and energy dissipation). It is an important step toward the actual use of such nonlinear simulation methods in industry. However, it may be recalled that these conclusions are only valuable for the proposed test structure "Harmony" for which all the contacts experience gross slip during vibration. In case of more complex behaviors at frictional interface such as microslip, a more accurate contact model would be necessary for the friction joint. Similarly, in some cases, a contact interface has to be described in details, with several nodes, in order 


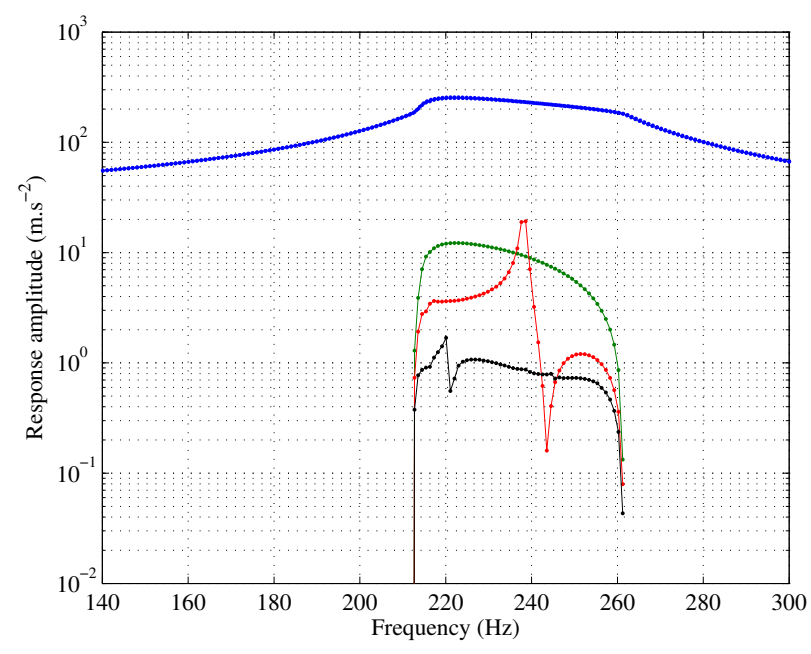

(a)

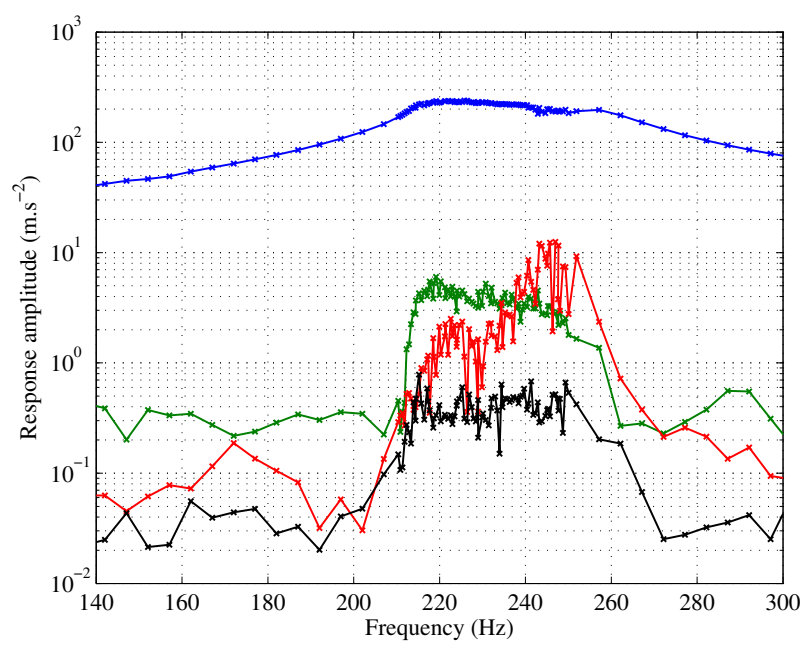

(b)

Figure 21: Simulated response (a) and measurements (b) at the top of the central body, with a 2910N excitation level, on different harmonics: $1^{\text {st }}$ (blue), $3^{\text {rd }}$ (green), $5^{\text {th }}$ (red) and $7^{\text {th }}$ (black).

to reproduce experimental results. Modeling and detailed understanding of the behavior at interfaces remain the key factors for a fair and reliable prediction of the vibration behavior of mechanical systems with frictional interfaces.

\section{Acknowledgement}

The authors gratefully acknowledge the support of the CEA/CESTA teams that helped with this study. They acknowledge in particular the experimental service who provided the measures presented in this paper.

J-J. Sinou acknowledges the support of the Institut Universitaire de France.

\section{References}

[1] K. Worden and G.R. Tomlinson. Nonlinearity in structural synamics: detection, identification and modelling. Institute of Physics Publishing, 2001.

[2] J. Warminski, S. Lenci, M.P. Carmtmell, G. Rega, and M. Wiercigroch. Nonlinear dynamic phenomena in mechanics. Springer, 2012.

[3] A.H. Nayfeh and D.T. Mook. Nonlinear oscillations. John Wiley \& Sons, 1979.

[4] A.F. Vakakis, L.I. Manevitch, Y.V. Mikhlin, V.N. Pilipchuck, and A.A. Zevin. Normal modes and localization in nonlinear systems. John Wiley \& Sons, 1996. 


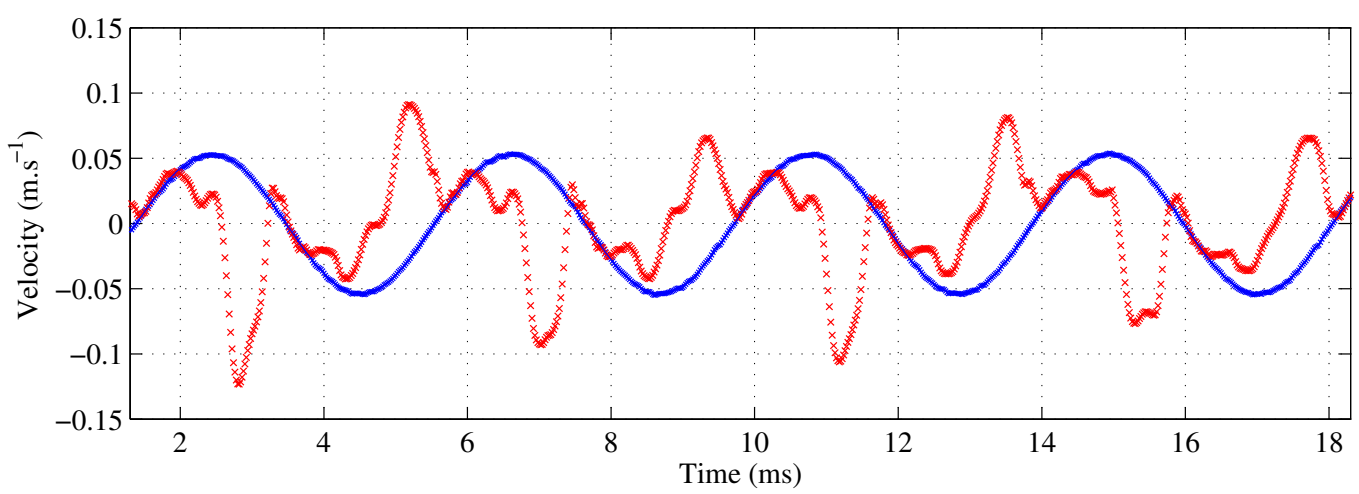

Figure 22: Measured velocities in the contact zone. The movement of the envelope (blue) and the top of the blade (red) are measured using the laser vibrometer.

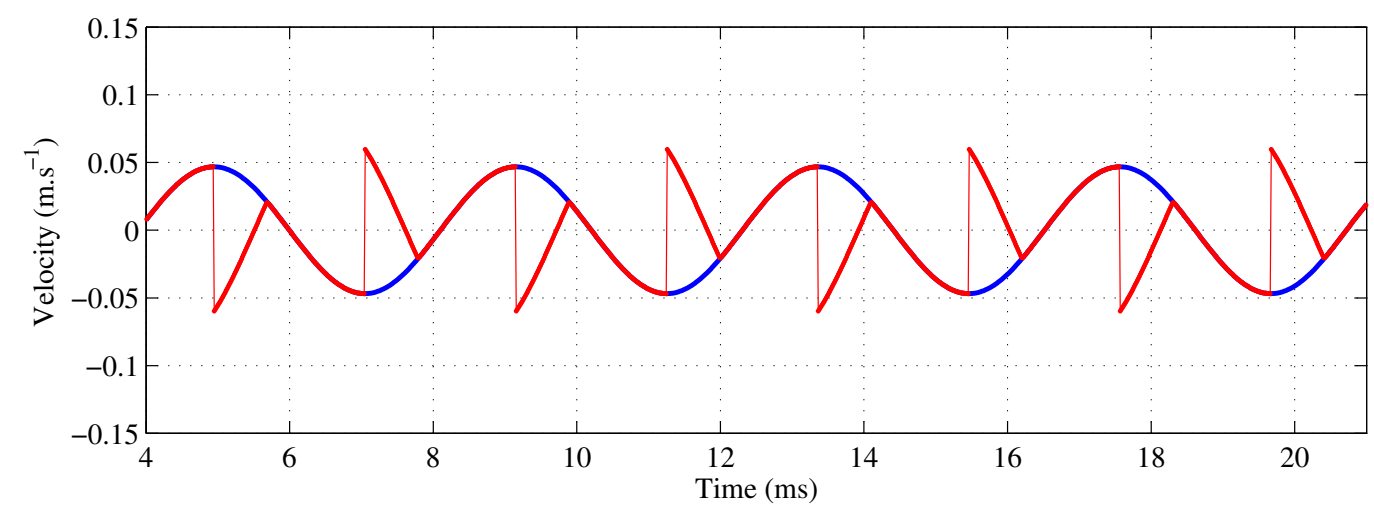

Figure 23: Simulated velocities in the contact zone using the 2-nodes Jenkins model presented in figure 18. The movement points $\mathrm{B}$ (in blue) and $\mathrm{P}$ (in green) are plotted.

[5] G. Kerschen, M. Peeters, J-C. Golinval, and A.F. Vakakis. Nonlinear normal modes. part 1: A useful framework for the structural dynamicist. Mechanical Systems and Signal Processing, 23:170-194, 2009.

[6] E.Sarrouy and J-J. Sinou. Chapter 21: Non-linear periodic and quasi-periodic vibrations in mechanical systems - On the use of the harmonic balance methods, Book: Advances in Vibration Analysis Research. Farzad Ebrahimi, INTECH, Open Access Publisher, Croatia, 2011.

[7] M. Claeys, J-J. Sinou, J-P. Lambelin, and B. Alcoverro. Multi-harmonic measurements and numerical simulations of nonlinear vibrations of a beam with non-ideal boundary conditions. Communications in Nonlinear Science and Numerical Simulation, 19:4196-4212, 2014.

[8] E.P. Petrov and D.J. Ewins. Analytical formulation of friction interface elements for analysis of nonlinear multi-harmonic vibrations of bladed disks. ASME Journal of Turbomachinery, 
125(2)1:364-371, 2003.

[9] C.Pierre, A.A. Ferri, and E.H. Dowell. Multi-harmonic analysis of dry friction damped systems using an incremental harmonic balance method. Transactions of the ASME, 52:958-964, 1985.

[10] K.Y. Sanliturk and D.J. Ewins. Modelling two-dimensional friction contact and its application using harmonic balance method. Journal of Sound and Vibration, 193(2):511-523, 1996.

[11] B. Al Sayed, E. Chatelet, S. Baguet, and G. Jacquet-Richardet. Dissipated energy and boundary condition effects associated to dry friction on the dynamics of vibrating structures. Mechanism and Machine Theory, 46:479-491, 2011.

[12] D. Süß and K. Willner. Investigation of a jointed friction oscillator using the multiharmonic balance method. Mechanical Systems and Signal Processing, 52:73-87, 2015.

[13] O. Poudou and C. Pierre. Hybrid frequency time domain methods for the analysis of complex structural systems with dry friction damping. Collection of Technical Papers AIAA/ASME/ASCE/AHS/ASC Structures, Structural Dynamics and Materials Conference, Norfolk,USA, 1:111-124, 2003.

[14] S. Nacivet, C. Pierre, F. Thouverez, and L. Jezequel. A dynamic lagrangian frequency-time method for the vibration of dry-friction-damped systems. Journal of Sound and Vibration, 265(1):201-219, 2003.

[15] K. Popp, L. Panning, and W. Sextro. Vibration damping by friction forces: Theory and applications. Journal of Vibration and Control, 9(3-4):419-448, 2003.

[16] A.V. Srinivasan and D.G. Cutts. Dry friction damping mechanisms in engine blades. Journal of Engineering for Power, 105(2):332-341, 1983.

[17] K.Y. Sanliturk, D.J. Ewins, and A.B. Stanbridge. Underplatform dampers for turbine blades: theoretical modeling, analysis, and comparison with experimental data. Journal of Engineering for Gas Turbines and Power, 123(4):919-929, 2006.

[18] I.A. Sever, E. Petrov, and D.J. Ewins. Experimental and numerical investigation of rotating bladed disk forced response using under-platform friction dampers. Proceeding of ASME Turbo Expo 200\%: Power for Land, Sea and Air, May 14-17, Montreal, Canada, Paper GT2007-27307, 2007.

[19] C.M. Firrone, D. Botto, and M.M. Gola. Optimization of interblade friction damper design. Proceeding of 8th Biennial ASME Conference on Engineering Systems Design and Analysis, July 4-7, Torino, Italy, Paper ESDA2006-95605:469-478, 2006.

[20] K.H. Koh, J.H. Griffin, S. Filippi, and A. Akay. Characterization of turbine blade friction dampers. Journal of Engineering for Gas Turbines and Power, 127(4):856-862, 2005. 
[21] W. Sextro. The calculation of the forced response of shrouded blades with friction contacts and its experimental verification. Proceedings of the ASME Turbo Expo 2000: Power for Land, Sea and Air, May 8-11, Munich, Germany, Paper No. 2000-GT-0540:1-8, 2000.

[22] L. Panning, W. Sextro, and K. Popp. Optimization of interblade friction damper design. Proceedings of the ASME Turbo Expo 2000: Power for Land, Sea and Air, May 8-11, Munich, Germany, Paper No. 2000-GT-0541:1-8, 2000.

[23] D. Charleux, C. Gibert, F. Thouverez, and J. Dupeux. Numerical and experimental study of friction damping in blade attachments of rotating bladed disks. International Journal of Rotating Machinery, Article ID 71302:1-13, 2006.

[24] D. Laxalde, C. Gibert, and F. Thouverez. Experimental and numerical investigation of friction rings damping of blisks. Proc. of ASME Turbo Expo 2008: Power for Land, Sea and Air, GT2008, June 9-13, Berlin, Germany, Paper GT2008-50862, 2008.

[25] L. Gaul and R. Nitsche. The role of friction in mechanical joints. Applied Mechanics Reviews, 54(2):93-106, 1998.

[26] B.F. Feeny, A. Guran, N. Hinrichs, and K. Popp. A historical review of dry friction and stick slip phenomena. Applied Mechanics Reviews, 51(5):321-341, 1998.

[27] T. Lauwagie, J. Guggenberger, J. Strobbe, and E. Dascotte. Model updating using operational data. In Proceedings of ISMA2010 including USD2010, pages 2669-2678, 2010.

[28] R.R. Craig and M.C.C. Bampton. Coupling of substructure for dynamic analysis. AIAA Journal, 1(2):1313-1319, 1968.

[29] M. Kim, V. Belsky, and M. Belyi. Substructure generation using automated multilevel substructuring, 2013. Patent US 2013/0124150A1 and EP 2597578A1.

[30] S. Zucca and B.I. Epureanu. Bi-linear reduced-order models of structures with friction intermittent contacts. Nonlinear Dynamics, 77:1055-1067, 2014.

[31] P.R. Dahl. Solid friction damping of mechanical vibrations. AIAA Journal, 14(12):1675-1682, 1976.

[32] W.D. Iwan. A distributed-element model for hysteresis and its steady-state dynamic response. Journal of Applied Mechanics, 14(12):893-900, 1966.

[33] L. Gaul and J. Lenz. Nonlinear dynamics of structures assembled by bolted joints. Acta Mechanica, 125(1-4):169-181, 1998.

[34] J. Guillen and C. Pierre. An efficient, hybrid, frequency-time domain method for the dynamics of large-scale dry-friction damped structural systems. In IUTAM Symposium on Unilateral Multibody Contacts, pages 169-178. Springer, 1999. 
[35] J-J. Sinou. Non-linear dynamics and contacts of an unbalanced flexible rotor supported on ball bearings. Mechanism and Machine Theory, 44:1713-1732, 2009.

[36] T.M. Cameron and J.H. Griffin. An alternating frequency time domain method for calculating the steady state response of nonlinear dynamic systems. ASME Journal of Applied Mechanics, 56:149-154, 1989 .

[37] O. Poudou. Modeling and analysis of the dynamics of dry-friction-damped structural systems. PhD Thesis, The University of Michigan, 2007. 